\title{
A thermo-chemo-mechanically coupled model for cathode particles in lithium-ion batteries
}

Received: 21 October 2020 / Revised: 27 January 2021 / Accepted: 5 March 2021 / Published online: 26 May 2021 (C) The Author(s) 2021

\begin{abstract}
As the demand for lithium-ion batteries increases, a better understanding of the complex phenomena involved in their operation becomes crucial. In this work, we propose a coupled thermo-chemo-mechanical model for electrode particles of $\mathrm{Li}$-ion batteries. To this end, we start with a general finite strain continuum framework for the coupled thermo-chemo-mechanical problem and then narrow it down to cathode active particles of Li-ion batteries, particularly to lithium manganese oxide particles. Electrochemical kinetics at the surface of the particle and also heat generation due to current exchange are taken into account. Next, the numerical treatment of the problem using the finite element method is presented. Specific line elements are needed to evaluate the flux of ions at the surface of the particle. Finally, the performance of the proposed model is evaluated using a few representative boundary value problems.
\end{abstract}

\section{Introduction}

Lithium-ion batteries are broadly used in portable consumer electronics, medical equipment, military devices, etc. Recently, the demand for lithium-ion batteries has increased dramatically due to the rise of electric and hybrid electric vehicles. Additionally, Li-ion batteries have the potential to be used for storing renewable energy derived from intermittent energy sources such as wind and sunlight, see Zubi et al. [61] and Diouf and Pode [20]. In comparison to other rechargeable batteries such as lead-acid or nickel-based batteries, Li-ion batteries provide higher specific energy and cycle life, which explains their popularity; however, due to safety reasons, they require a battery management system. With the increase in the demand for Li-ion batteries, researchers continue to enhance their performance and safety while decreasing the production costs.

A Li-ion battery cell consists of four main components: an anode, a cathode, a separator and an electrolyte. The anode is typically made of graphite, the cathode is normally a lithium metal oxide and the electrolyte is usually a lithium salt mixed in an organic solvent. The separator, which is usually made of polyethylene or polypropylene, has almost zero electrical conductivity and is placed between the electrodes to prevent short circuit while allowing the flow of lithium ions through its micropores. Despite the attempts to improve the battery capacity by replacing graphite anodes with alternative materials such as silicon-based anodes, graphite remains the preferred anode material for most industrial applications. Hence, the cathode active materials have been the focus of attention in enhancing battery performance. Some examples of cathode active materials are: lithium cobalt oxide ( $\mathrm{LiCo} \mathrm{O}_{2}$ or $\left.\mathrm{LCO}\right)$, lithium manganese oxide $\left(\mathrm{Li} \mathrm{Mn}_{2} \mathrm{O}_{4}\right.$ or $\left.\mathrm{LMO}\right)$, lithium nickel manganese cobalt oxide (NMC) and lithium nickel cobalt aluminum oxide (NCA), see Buchmann [8] for a lucid introduction. The reader is also referred to Whittingham [52]. The latter two cathode chemistries mentioned above have risen in popularity in the recent years, especially in electric vehicles. The charging process in Li-ion batteries is driven by an external voltage. During the charging of the battery, electrons move in the 
outer circuit from cathode to anode. In the meanwhile, lithium ions are extracted from the cathode, migrate in the electrolyte, permeate the separator and intercalate into the anode. During discharging, the process is reversed and the generated electric current in the outer circuit can be exploited. Charging and discharging of battery, and hence, intercalation and extractions of lithium ions, cause volumetric deformation of electrode active materials. The resulting stresses may lead to fracture of the particles of active materials, and thus, to battery capacity fade, see Wang et al. [48], [49] and also the review article of Lyu et al. [34].

The growing demand for $\mathrm{Li}$-ion batteries has risen questions about sustainability and environmental impacts of their extensive use. In addition to these issues, there remain some technical challenges for engineers in the application of Li-ion batteries, for example, capacity fade, self-discharge, thermal runaway and poor low temperature performance, see Bandhauer et al. [3] for a good review and the references cited therein. All of these issues are linked with thermal effects in the battery. Of all the issues listed above, thermal runaway is probably the most critical for electric vehicles as it poses a threat to the safety of the passengers. Thermal runaway occurs when a chain of unwanted exothermic reactions takes place in the battery, which can eventually lead to battery failure, venting with flames and even explosion, see Feng et al. [23] and Yang et al. [54] for a description of possible mechanisms of thermal runaway.

An interplay of complex effects across multiple scales, which influence each other directly or indirectly, is observed in Li-ion batteries. A complete picture of these effects may be achieved by considering the coupling between electrochemistry, thermodynamics and mechanics. Pioneering efforts to model full lithium battery cells mathematically were made by Doyle et al. [21] for solid-state batteries and Fuller et al. [24] for Li-ion batteries. These rigorous one-dimensional models are purely electrochemical. A two-dimensional model for evaluation of electrochemical fields and chemically induced stresses within porous cathodes was proposed by García et al. [25] based on a linearized form of the Butler-Volmer interfacial kinetics. Christensen and Newman [13] proposed a continuum model for intercalation-induced stress generation in spherical particles of electrode active materials. This model was then used in Christensen and Newman [14] to predict fracture onset in spherical lithium manganese oxide particles. A three-dimensional model for predicting intercalation-induced stresses within cathode particles was proposed by Zhang et al. [56]. This model was utilized in the subsequent work of Zhang et al. [57], which additionally took electrochemical kinetics into account using the ButlerVolmer equation. The latter model could, furthermore, predict intercalation-induced heat generation. The authors, however, did not comment on the numerical implementation of their model. Analytical relationships for diffusion-induced stresses in spherical electrode particles were proposed in Cheng and Verbrugge [10-12] and Verbrugge and Cheng [47]. Several other models have been proposed which treat phase transformation [6, 17,42-44,50,59], fracture [1,18,19,30-32], plasticity [7,58], homogenization [26] and structural instabilities [53] in Li-ion batteries, just to name a few. See also the review article by Zhao et al. [60] on coupled chemomechanical modeling approaches for $\mathrm{Li}$-ion batteries across various scales.

Predicting the amount of heat generation during the operation of batteries is of key importance in designing thermal management systems. Numerous efforts have been made to predict and simulate heat generation in batteries. A general expression for energy balance in battery cells, which considered electrochemical reactions, phase transitions, mixing effects and Joule heating, was given in Bernardi et al. [4]. In their work, the temperature inside the cell was assumed to be uniform. Gu and Wang [27] presented a coupled thermal and electrochemical model using the volume-averaging approach, which allowed for a spatial variation of temperature within the battery cell. Heat generation in a one-dimensional cell with a porous electrode was simulated by Thomas and Newman [45] and compared with the experimental measurements. A three-dimensional thermal model for batteries with layered structure was given in Chen et al. [9]. Zhang et al. [57] used the expression given by Thomas and Newman [45] to calculate heat generation in LMO cathode particles under potentio-dynamic control. A different formulation of transport phenomena in Li-ion batteries, including heat transfer, was given by Latz and Zausch [33] based on non-equilibrium thermodynamics, see De Groot and Mazur [16]. See also the work of $\mathrm{Hu}$ and Shen [29] on variational formulation of coupled thermo-chemo-mechanical processes based on non-equilibrium thermodynamics. Hu et al. [28] presented a continuum model for hollow core-shell electrodes by coupling a one-dimensional electrochemical battery model with a two-dimensional thermal model. Werner and Weinberg [51] proposed, based on thermodynamics of irreversible processes, a coupled thermo-chemo-mechanical model for solid state batteries, which also takes phenomena such as phase transformations, thermal diffusion and thermoelectric effects into account. A coupled thermo-chemomechanical model for graphite, NMC, and LMO electrode particles was proposed by Masmoudi et al. [35] based on thermodynamics of irreversible processes. Their small strain formulation has been supplemented with numerical examples; however, an algorithmic implementation of the model has not been addressed. 
Following the previous works by Dal and Miehe [15] and Miehe et al. [38], we propose a coupled thermochemo-mechanical model for electrode particles in Li-ion batteries. Our four-field formulation uses also elements of the previous works by Miehe et al. [36] on variational formulation of diffusion in elastic solids and Miehe et al. [37] on phase field modeling of brittle fracture in thermo-elastic solids. The model is used to simulate the response of LMO cathode particles during battery operation. This finite strain model can be used to simulate other electrode particles, provided that the electrochemical properties of the material and the necessary material parameters are known. In Sect. 2 of this work, a general framework for coupled thermo-chemo-mechanics is formulated. The governing equations of the coupled problem are given and the thermodynamical consistency of the model is discussed. An alternative approach to fulfill the second law of thermodynamics is discussed, which is related in the subsequent section of the paper to Onsager's reciprocal relations. In Sect. 3, the general framework of Sect. 2 is narrowed down to electrode particles of Li-ion batteries. To this end, the corresponding constitutive functions are specified and the electrochemical reaction kinetics and heat generation at the boundary of particles are discussed. In Sect. 4, the numerical implementation of the model with a specific application to electrode particles is presented. Finally, in Sect. 5, the model response is evaluated with the help of a few representative boundary value problems.

\section{Four-field formulation of the coupled problem}

This section outlines a general continuum formulation of coupled thermo-chemo-mechanics. We will start by introducing the primary field variables, their gradients and the material and spatial fluxes. We will then discuss the global and local balance laws of the coupled problem, the principle of irreversibility and the constitutive equations that fulfill the principle of irreversibility.

\subsection{Primary field variables of the coupled problem}

The coupled problem under consideration can be treated as a four-field problem. The primary field variables are the deformation of the solid

$$
\boldsymbol{\varphi}:\left\{\begin{array}{l}
\mathcal{B}_{0} \times \mathbb{R}^{+} \rightarrow \mathcal{B}_{t} \subset \mathbb{R}^{3} \\
(X, t) \mapsto x=\varphi(X, t),
\end{array}\right.
$$

the species concentration and the chemical potential

$$
c:\left\{\begin{array}{l}
\mathcal{B}_{0} \times \mathbb{R}^{+} \rightarrow[0,1] \\
(\boldsymbol{X}, t) \mapsto c(\boldsymbol{X}, t)
\end{array} \quad \text { and } \quad \mu:\left\{\begin{array}{l}
\mathcal{B}_{0} \times \mathbb{R}^{+} \rightarrow \mathbb{R} \\
(\boldsymbol{X}, t) \mapsto \mu(\boldsymbol{X}, t)
\end{array}\right.\right.
$$

and the absolute temperature

$$
\theta: \begin{cases}\mathcal{B}_{0} \times \mathbb{R}^{+} & \rightarrow \mathbb{R}^{+} \\ (\boldsymbol{X}, t) & \mapsto \theta(\boldsymbol{X}, t)\end{cases}
$$

At any time $t \in \mathbb{R}^{+}$, the deformation $\varphi$ is a one-to-one mapping of the points $X \in \mathcal{B}_{0}$ of the reference configuration $\mathcal{B}_{0}$ onto the points $\boldsymbol{x} \in \mathcal{B}_{t}$ of the current configuration $\mathcal{B}_{t}$. The concentration field $c \in[0,1]$ is a dimensionless measure of the amount of the diffusing species (lithium ions) in unit reference volume of the solid. It may be considered as the molar concentration $\tilde{c}$ of the diffusing species in unit reference volume of the solid divided by the maximum possible molar concentration $\tilde{c}_{\max }$. The chemical potential $\mu$ is the energy-conjugate variable to concentration. The absolute temperature $\theta$ is a positive scalar field defined over $\mathcal{B}_{0}$, i.e., $\theta \geq 0$. See Fig. 1 for a visualization of the primary variables. The material gradients of deformation, (negative) chemical potential and temperature fields are denoted by $\boldsymbol{F}, \mathbb{M}$ and $\mathbb{F}$ and defined by

$$
\boldsymbol{F}:=\nabla \varphi(\boldsymbol{X}, t), \quad \mathbb{M}:=-\nabla \mu(\boldsymbol{X}, t), \quad \mathbb{F}:=\nabla \theta(\boldsymbol{X}, t) .
$$

In order to circumvent penetration of matter, we require that the determinant of the deformation gradient be positive, i.e., $J:=\operatorname{det}[\boldsymbol{F}]>0$. The deformation gradient $\boldsymbol{F}$, its cofactor $\operatorname{cof}[\boldsymbol{F}]=J \boldsymbol{F}^{-T}$ and its determinant $J$ serve as the mappings of infinitesimal line, area and volume elements, respectively:

$$
\mathrm{d} \boldsymbol{x}=\boldsymbol{F} \mathrm{d} \boldsymbol{X}, \quad \mathrm{d} \boldsymbol{a}=\operatorname{cof}[\boldsymbol{F}] \mathrm{d} \boldsymbol{A}, \quad \mathrm{d} v=J \mathrm{~d} V .
$$



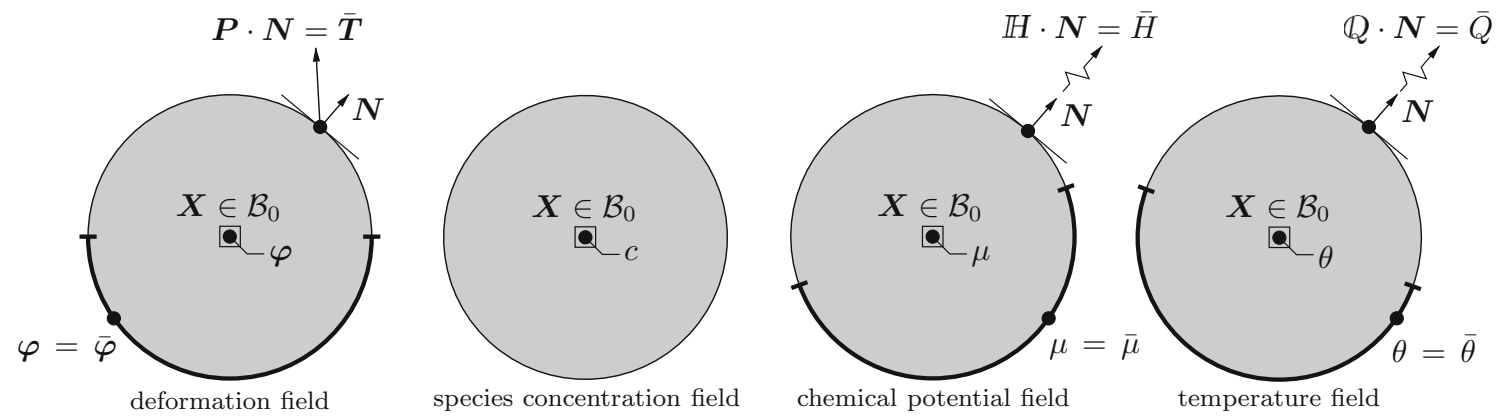

Fig. 1 Primary field variables of the coupled thermo-chemo-mechanical problem. The boundary $\partial \mathcal{B}_{0}$ of the reference configuration is decomposed into Dirichlet and Neumann boundaries for the deformation field $\varphi$, the chemical potential field $\mu$ and the temperature field $\theta$. The prescribed values on the boundary are marked with an overbar

\subsection{Stress tensors and flux vectors}

Consider an arbitrary part $\mathcal{P}_{0} \subset \mathcal{B}_{0}$ cut out of the reference configuration $\mathcal{B}_{0}$ of the body under consideration. Let $\mathcal{P}_{t} \subset \mathcal{B}_{t}$ denote the deformed configuration of $\mathcal{P}_{0}$ at time $t$. Thus, we can write $\mathcal{P}_{t}=\varphi_{t}\left(\mathcal{P}_{0}\right)$. Let also $\partial \mathcal{P}_{0}$ and $\partial \mathcal{P}_{t}$ express the boundaries of $\mathcal{P}_{0}$ and $\mathcal{P}_{t}$, respectively. We will write the balance laws for this arbitrary part, assuming that solid material can not cross the boundary $\partial \mathcal{P}_{t}$ of the spatial region $\mathcal{P}_{t}$ as it deforms with the body.

\subsubsection{Stress tensors}

The mechanical force per unit area exerted on $\mathcal{P}_{t}$ by the rest of the body $\mathcal{B}_{t} \backslash \mathcal{P}_{t}$ is called the traction vector and is denoted by $\boldsymbol{t}$. Based on Cauchy's stress theorem, at any point $\boldsymbol{x} \in \partial \mathcal{P}_{t}$ there exists a second-order tensor $\sigma$, called the Cauchy stress tensor, that relates the outward unit normal $\boldsymbol{n}$ to the traction vector $\boldsymbol{t}$ acting on $\mathcal{P}_{t}$ :

$$
\boldsymbol{t}(\boldsymbol{x}, t ; \boldsymbol{n})=\sigma(\boldsymbol{x}, t) \cdot \boldsymbol{n} .
$$

We define the referential traction vector $\boldsymbol{T}$ by scaling the spatial force $t \mathrm{~d} a$ to the referential area element $d A$ such that the identity $\boldsymbol{t} \mathrm{d} a=\boldsymbol{T} \mathrm{d} A$ holds. The first Piola-Kirchhoff stress tensor $\boldsymbol{P}$ is then defined by forming a relationship, analogous to (6), between the referential outward unit normal $\boldsymbol{N}$ and traction vector $\boldsymbol{T}$ :

$$
\boldsymbol{T}(\boldsymbol{X}, t ; \boldsymbol{N})=\boldsymbol{P}(\boldsymbol{X}, t) \cdot \boldsymbol{N} .
$$

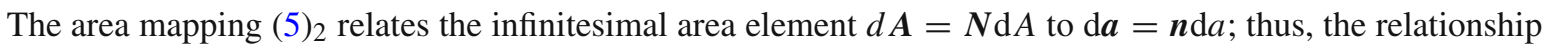
between the first Piola-Kirchhoff stress tensor $\boldsymbol{P}$ and the Cauchy stress tensor $\boldsymbol{\sigma}$ can be obtained as

$$
\boldsymbol{P}=J \boldsymbol{\sigma} \boldsymbol{F}^{-T} .
$$

\subsubsection{Species flux vectors}

The species flux $h$ represents the amount of the species that flows outwards, per unit time, through a unit area element of the boundary $\partial \mathcal{P}_{t}$ of the part $\mathcal{P}_{t}$. It is related to the outward normal $\boldsymbol{n}$ by a flux vector $\mathbb{h}$ in the form

$$
h(\boldsymbol{x}, t ; \boldsymbol{n})=\mathbb{h}(\boldsymbol{x}, t) \cdot \boldsymbol{n} .
$$

The material species flux, denoted by $H$ and defined by the identity $h \mathrm{~d} a=H \mathrm{~d} A$, represents the flow of the species, per unit time, through a unit area element of the boundary $\partial \mathcal{P}_{0}$. In analogy to (9), we assume that the material species flux $H$ is related to the outward normal $N$ to $\partial \mathcal{P}_{0}$ by a material species flux vector $\mathbb{H}$

$$
H(X, t ; N)=\mathbb{H}(\boldsymbol{X}, t) \cdot \boldsymbol{N} .
$$

Using the area mapping $(5)_{2}$, the relationship between the material species flux vector $\mathbb{H}$ and the spatial species flux vector th is obtained:

$$
\mathbb{H}=J \boldsymbol{F}^{-1} \mathfrak{h} .
$$




\subsubsection{Heat flux vectors}

The heat flux $q$ is the outward flow of thermal energy, per unit time, through a unit area element of the boundary $\partial \mathcal{P}_{t}$. It is related to the outward normal $\boldsymbol{n}$ to the surface $\partial \mathcal{P}_{t}$ by the heat flux vector $\mathscr{q}$

$$
q(\boldsymbol{x}, t ; \boldsymbol{n})=q(\boldsymbol{x}, t) \cdot \boldsymbol{n} .
$$

The material heat flux $Q$ is defined by the identity $q \mathrm{~d} a=Q \mathrm{~d} A$ and represents the amount of thermal energy which flows outward, per unit time, through a unit area element of the boundary $\partial \mathcal{P}_{0}$. Similar to (12), the material heat flux $Q$ is related to the outward normal $N$ to $\partial \mathcal{P}_{0}$ by a material heat flux vector $\mathbb{Q}$

$$
Q(X, t ; N)=\mathbb{Q}(X, t) \cdot N .
$$

With the area mapping $(5)_{2}$, the relationship between the material heat flux vector $\mathbb{Q}$ and the spatial heat flux vector $\mathbb{q}$ can be written as

$$
\mathbb{Q}=J \boldsymbol{F}^{-1} \mathbb{q}
$$

\subsection{General equations of thermo-chemo-mechanics}

\subsubsection{Global balance equations}

The general equations governing the coupled problem are written for the arbitrary part $\mathcal{P}_{0} \subset \mathcal{B}_{0}$ cut out of the reference configuration $\mathcal{B}_{0}$. No mass production is assumed to take place. During the deformation the total mass of the solid constituent in the part $\mathcal{P}_{0}$ should remain unchanged and equal to its initial value. Considering the part $\mathcal{P}_{0}$ as a control volume, balance of species content demands that the temporal changes in the amount of the diffusing species be equal to the in-flux (negative out-flux) through the surface

$$
\frac{\mathrm{d}}{\mathrm{d} t} \int_{\mathcal{P}_{0}} c \mathrm{~d} V=-\int_{\partial \mathcal{P}_{0}} H \mathrm{~d} A .
$$

Neglecting the inertial effects, balances of linear and angular momentum for the part $\mathcal{P}_{0}$ can be written as

$$
\int_{\partial \mathcal{P}_{0}} \boldsymbol{T} \mathrm{d} A+\int_{\mathcal{P}_{0}} \gamma \mathrm{d} V=\mathbf{0} \quad \text { and } \quad \int_{\partial \mathcal{P}_{0}} \boldsymbol{\varphi} \times \boldsymbol{T} \mathrm{d} A+\int_{\mathcal{P}_{0}} \boldsymbol{\varphi} \times \boldsymbol{\gamma} \mathrm{d} V=\mathbf{0},
$$

respectively. In the above equations, $\boldsymbol{\gamma}(\boldsymbol{X}, t)$ is the body force exerted per unit reference volume. Finally, the balance of energy reads

$$
\frac{\mathrm{d}}{\mathrm{d} t} \int_{\mathcal{P}_{0}} e \mathrm{~d} V=\int_{\partial \mathcal{P}_{0}}(\boldsymbol{T} \cdot \boldsymbol{V}-Q-\mu H) \mathrm{d} A+\int_{\mathcal{P}_{0}}(\boldsymbol{\gamma} \cdot \boldsymbol{V}+r) \mathrm{d} V,
$$

where $\boldsymbol{V}(\boldsymbol{X}, t):=\dot{\boldsymbol{\varphi}}(\boldsymbol{X}, t)$ is the material velocity field, $e(\boldsymbol{X}, t)$ is the internal energy of the unit reference volume and $r(\boldsymbol{X}, t)$ is the heat source per unit reference volume.

\subsubsection{Local balance equations}

Using the definitions introduced in Sect. 2.2 for the material species flux $H$, the nominal traction vector $\boldsymbol{T}$ and the material heat flux $Q$ and with the help of the Gauss and localization theorems, we can write the local balance equations

\begin{tabular}{|lc|}
\hline 1. Balance of species content & $\dot{c}=-\operatorname{Div}[\mathbb{H}]$ \\
2. Balance of linear momentum & $\operatorname{Div}[\boldsymbol{P}]+\boldsymbol{\gamma}=\mathbf{0}$ \\
3. Balance of angular momentum & $\operatorname{skew}\left[\boldsymbol{P} \boldsymbol{F}^{T}\right]=\mathbf{0}$ \\
4. Balance of energy & $\dot{e}=\boldsymbol{P}: \dot{\boldsymbol{F}}-\operatorname{Div}[\mathbb{Q}]+\mu \dot{c}+r+\mathbb{H} \cdot \mathbb{M}$ \\
\hline
\end{tabular}




\subsubsection{Principle of irreversibility}

The global form of the second axiom of thermodynamics for an arbitrary part $\mathcal{P}_{0} \subset \mathcal{B}_{0}$ of the body is

$$
\frac{\mathrm{d}}{\mathrm{d} t} \int_{\mathcal{P}_{0}} \eta \mathrm{d} V \geq-\int_{\partial \mathcal{P}_{0}} \frac{Q}{\theta} \mathrm{d} A+\int_{\mathcal{P}_{0}} \frac{r}{\theta} \mathrm{d} V,
$$

where $\eta(\boldsymbol{X}, t)$ is the entropy per unit reference volume. The local form of the entropy imbalance reads

$$
\dot{\eta}-\frac{1}{\theta}(r-\operatorname{Div}[\mathbb{Q}])-\frac{1}{\theta^{2}} \mathbb{Q} \cdot \nabla \theta \geq 0 .
$$

Using the balance of energy $(18)_{4}$ and introducing the Helmholtz free energy $\psi$ by the Legendre transformation $e=\psi+\eta \theta$, the above inequality takes the form

$$
\dot{\eta}-\frac{1}{\theta}(\dot{\psi}+\dot{\eta} \theta+\eta \dot{\theta}-\boldsymbol{P}: \dot{\boldsymbol{F}}-\mu \dot{c}-\mathbb{H} \cdot \mathbb{M})-\frac{1}{\theta^{2}} \mathbb{Q} \cdot \nabla \theta \geq 0 .
$$

Multiplying the above inequality by the absolute temperature and defining $\mathbb{G}:=-\frac{\nabla \theta}{\theta}$, the local form of the second axiom is obtained in the form of the dissipation inequality

$$
\mathcal{D}:=\boldsymbol{P}: \dot{\boldsymbol{F}}-\eta \dot{\theta}+\mu \dot{c}-\dot{\psi}+\mathbb{H} \cdot \mathbb{M}+\mathbb{Q} \cdot \mathbb{G} \geq 0 .
$$

This poses a constraint on the material modeling framework. It needs to be fulfilled locally such that the second axiom of thermodynamics is not violated.

A stronger constraint is achieved by demanding the positiveness of some sets of the individual terms in the dissipation inequality. To this end, the dissipation inequality (22) is split up into an internal part and a part due to diffusion and heat conduction and each of these parts is demanded to be non-negative:

$$
\mathcal{D}_{\text {int }}:=\boldsymbol{P}: \dot{\boldsymbol{F}}-\eta \dot{\theta}+\mu \dot{c}-\dot{\psi} \geq 0 \quad ; \quad \mathcal{D}_{\text {dif,con }}:=\mathbb{H} \cdot \mathbb{M}+\mathbb{Q} \cdot \mathbb{G} \geq 0 .
$$

An even stronger constraint may be achieved by splitting the dissipation inequality (22) into an internal part and two separate parts due to diffusion and heat conduction. Each of these parts is demanded to be non-negative separately:

$$
\mathcal{D}_{\text {int }}:=\boldsymbol{P}: \dot{\boldsymbol{F}}-\eta \dot{\theta}+\mu \dot{c}-\dot{\psi} \geq 0 ; \quad \mathcal{D}_{\text {dif }}:=\mathbb{H} \cdot \mathbb{M} \geq 0 ; \quad \mathcal{D}_{\text {con }}:=\mathbb{Q} \cdot \mathbb{G} \geq 0 .
$$

\subsubsection{Objective free energy and thermodynamical forces}

In order to close the problem, we need to specify the constitutive functions such that the principle of irreversibility is fulfilled. Assuming a local theory of grade one, the Helmholtz free energy $\psi$ is assumed to depend on the primary variables $\boldsymbol{\varphi}, c$ and $\theta$ and their first gradients,

$$
\psi=\bar{\psi}(\boldsymbol{\varphi}, \nabla \boldsymbol{\varphi}, c, \nabla c, \theta, \nabla \theta) .
$$

The stored energy function $\bar{\psi}$ must remain invariant under a rigid body motion of the form $\boldsymbol{\varphi}^{+}=\boldsymbol{R} \boldsymbol{\varphi}+\boldsymbol{c}$ superimposed on the current configuration. Here, $\boldsymbol{R}(t) \in S O(3)$ denotes an arbitrarily chosen rotation tensor of the special orthogonal group and $\boldsymbol{c}(t)$ is a translation vector. Based on this requirement, the stored energy function $\bar{\psi}$ can not depend on $\boldsymbol{\varphi}$ and depends on the deformation gradient $\boldsymbol{F}$ through the right Cauchy-Green tensor $\boldsymbol{C}:=\boldsymbol{F}^{T} \boldsymbol{F}$, which remains invariant under this rigid body motion. This conclusion together with a standard Coleman-Noll procedure applied on the internal dissipation inequality $(23)_{1}$ or $(24)_{1}$ gives the reduced set of arguments for the stored energy

$$
\psi=\widehat{\psi}(\boldsymbol{F}, c, \theta)=\widetilde{\psi}(\boldsymbol{C}, c, \theta),
$$

and the constitutive equations for the thermodynamical forces

$$
\boldsymbol{P}=\partial_{\boldsymbol{F}} \widehat{\psi}(\boldsymbol{F}, c, \theta) ; \mu=\partial_{c} \widehat{\psi}(\boldsymbol{F}, c, \theta) ; \quad \eta=-\partial_{\theta} \widehat{\psi}(\boldsymbol{F}, c, \theta) .
$$

Note that by making the stored energy function dependent on the right Cauchy-Green tensor $\boldsymbol{C}$, the balance of angular momentum is automatically fulfilled. 


\subsubsection{Temperature evolution equation}

With $e=\psi+\eta \theta$ and the constitutive equations given in (27), balance of energy (18) 4 takes the form of a temperature evolution equation

$$
\mathbb{C} \dot{\theta}=-\operatorname{Div}[\mathbb{Q}]+\mathbb{H} \cdot \mathbb{M}+r+\mathcal{L},
$$

where the following definitions are used for the sake of compactness:

$$
\mathbb{C}:=-\theta \partial_{\theta \theta}^{2} \widehat{\psi} \quad \text { and } \quad \mathcal{L}:=\theta \partial_{\boldsymbol{F} \theta}^{2} \widehat{\psi}: \dot{\boldsymbol{F}}+\theta \partial_{c \theta}^{2} \widehat{\psi} \dot{c}
$$

\subsubsection{Thermo-chemically coupled dissipation potential}

A thermodynamically consistent setting can be achieved by demanding that the internal part of the dissipation inequality (23) $)_{1}$ and the part due to diffusion and heat conduction $(23)_{2}$ be non-negative. Let us repeat these inequalities:

$$
\mathcal{D}_{\text {int }}:=\boldsymbol{P}: \dot{\boldsymbol{F}}-\eta \dot{\theta}+\mu \dot{c}-\dot{\psi} \geq 0 \quad ; \quad \mathcal{D}_{\text {dif,con }}:=\mathbb{H} \cdot \mathbb{M}+\mathbb{Q} \cdot \mathbb{G} \geq 0 .
$$

Following a standard Coleman-Noll procedure and using the constitutive equation (27), the internal dissipation inequality $(30)_{1}$ can a-priori be fulfilled. What remains is to restrict the material modeling framework such that the dissipation due to diffusion and heat conduction $(30)_{2}$ is also non-negative. Let us define the combined flux vector $\mathcal{J}:=[\mathbb{H} ; \mathbb{Q}]$ and the combined driving force $\mathcal{I}:=[\mathbb{M} ; \mathbb{G}]$. The dissipation inequality due to diffusion and heat conduction $(30)_{2}$ takes the form

$$
\mathcal{D}_{\text {dif,con }}:=\mathcal{J} \cdot \mathcal{I} \geq 0 .
$$

We assume that the flux vector $\mathcal{J}$ is specified by an objective dissipation potential

$$
\phi=\widehat{\phi}(\mathcal{I} ; \boldsymbol{F}, c, \theta)=\widetilde{\phi}(\mathcal{I} ; \boldsymbol{C}, c, \theta),
$$

which depends on the driving force $\mathcal{I}$ at a given state $\{\boldsymbol{F}, c, \theta\}$ of deformation, concentration and temperature. For a convex, positive and normalized $\phi$, the dissipation inequality (31) is a-priori fulfilled if the flux vector $\mathcal{J}$ is specified by the following constitutive equation:

$$
\mathcal{J}=\partial_{\mathcal{I}} \widehat{\phi}(\mathcal{I} ; \boldsymbol{F}, c, \theta)
$$

Convexity of the dissipation potential in $\mathcal{I}$ implies that the Hessian $\mathfrak{H}:=\partial_{\mathcal{I} \mathcal{I}}^{2} \widehat{\phi}$ is positive semi-definite. Moreover, $\mathfrak{H}$ is a symmetric tensor due to symmetry of second derivatives. Let us divide $\mathfrak{H}$ into four blocks as below:

$$
\mathfrak{H}=\left[\begin{array}{ll}
\partial_{\mathbb{M M}}^{2} \widehat{\phi} & \partial_{\mathbb{M} G}^{2} \widehat{\phi} \\
\partial_{\mathbb{G M}}^{2} & \partial_{\mathbb{G} \mathbb{G}}^{2} \widehat{\phi}
\end{array}\right]=:\left[\begin{array}{ll}
\mathfrak{a} & \mathfrak{b} \\
\mathfrak{b}^{T} & \mathfrak{c}
\end{array}\right],
$$

where we $\mathfrak{a}$ and $\mathfrak{c}$ are themselves symmetric. Positive semi-definiteness of $\mathfrak{H}$ implies a constraint on the blocks $\mathfrak{a}, \mathfrak{b}$ and $\mathfrak{c}$. For a positive semi-definite block $\mathfrak{a} \succeq 0$, the tensor given in (34) is positive semi-definite if and only if the Schur complement $\mathfrak{H} / \mathfrak{a}$ of $\mathfrak{a}$ is also positive semi-definite, that is

$$
\mathfrak{H} / \mathfrak{a}:=\mathfrak{c}-\mathfrak{b}^{T} \mathfrak{a}^{-1} \mathfrak{b} \succeq 0 .
$$

With (27) and (33) at hand, we can summarize the constitutive equations in the following box:

\begin{tabular}{|lrl}
\hline 1. Constitutive stress & $\boldsymbol{P}$ & $=\partial_{\boldsymbol{F}} \widehat{\psi}(\boldsymbol{F}, c, \theta)$ \\
2. Constitutive chemical potential $\mu$ & $=\partial_{c} \widehat{\psi}(\boldsymbol{F}, c, \theta)$ \\
3. Constitutive entropy & $\eta$ & $=-\partial_{\theta} \widehat{\psi}(\boldsymbol{F}, c, \theta)$ \\
4. Constitutive flux vector & $\mathcal{J}$ & $=\partial_{\mathcal{I}} \widehat{\phi}(\mathcal{I} ; \boldsymbol{F}, c, \theta)$ \\
\hline
\end{tabular}




\subsubsection{Decoupled chemical and thermal dissipation potentials}

A simpler formulation with decoupled thermal and chemical dissipation potentials is obtained by demanding that the dissipation inequalities (24) be satisfied. Let us repeat these inequalities:

$$
\mathcal{D}_{\text {int }}:=\boldsymbol{P}: \dot{\boldsymbol{F}}-\eta \dot{\theta}+\mu \dot{c}-\dot{\psi} \geq 0 \quad ; \quad \mathcal{D}_{\text {dif }}:=\mathbb{H} \cdot \mathbb{M} \geq 0 \quad ; \quad \mathcal{D}_{\text {con }}:=\mathbb{Q} \cdot \mathbb{G} \geq 0 .
$$

Following a standard Coleman-Noll procedure and making use of the constitutive Eq. (27), the internal dissipation inequality (37) 1 can a-priori be fulfilled. We need to restrict the material modeling framework such that the dissipation inequalities due to diffusion $(37)_{2}$ and heat conduction $(37)_{3}$ are also satisfied. To this end, the material species flux vector $\mathbb{H}$ is assumed to be specified by the objective chemical dissipation potential

$$
\phi_{c}=\widehat{\phi}_{c}(\mathbb{M} ; \boldsymbol{F}, c, \theta)=\widetilde{\phi}_{c}(\mathbb{M} ; \boldsymbol{C}, c, \theta),
$$

which depends on the (negative) gradient $\mathbb{M}$ of chemical potential at a given state $\{\boldsymbol{F}, c, \theta\}$ of deformation, concentration and temperature. Assuming a convex, positive and normalized chemical dissipation potential $\phi_{c}$ in terms of $\mathbb{M}$, the dissipation inequality $(37)_{2}$ is a-priori satisfied if the material species flux vector $\mathbb{H}$ is specified by the constitutive equation

$$
\mathbb{H}=\partial_{\mathbb{M}} \widehat{\phi}_{c}(\mathbb{M} ; \boldsymbol{F}, c, \theta) .
$$

Similarly, we assume the material heat flux vector $\mathbb{Q}$ to be specified by an objective thermal dissipation potential

$$
\phi_{t}=\widehat{\phi}_{t}(\mathbb{G} ; \boldsymbol{F}, c, \theta)=\widetilde{\phi}_{t}(\mathbb{G} ; \boldsymbol{C}, c, \theta),
$$

which depends on the vector $\mathbb{G}$ at a given state $\{\boldsymbol{F}, c, \theta\}$ of deformation, concentration and temperature. For a convex, positive and normalized $\phi_{t}$, the dissipation inequality $(37)_{3}$ is a-priori fulfilled if the material heat flux vector $\mathbb{Q}$ is specified by the following constitutive equation:

$$
\mathbb{Q}=\partial_{\mathbb{G}} \widehat{\phi}_{t}(\mathbb{G} ; \boldsymbol{F}, c, \theta) .
$$

With (27), (39) and (41) at hand, we can summarize the constitutive equations in the following box:

$\begin{array}{|lrl|}\text { 1. Constitutive stress } & \boldsymbol{P} & =\partial_{\boldsymbol{F}} \widehat{\psi}(\boldsymbol{F}, c, \theta) \\ \text { 2. Constitutive chemical potential } \mu & =\partial_{c} \widehat{\psi}(\boldsymbol{F}, c, \theta) \\ \text { 3. Constitutive entropy } & \eta=-\partial_{\theta} \widehat{\psi}(\boldsymbol{F}, c, \theta) \\ \text { 4. Constitutive species flux vector } \mathbb{H}=\partial_{\mathbb{M}} \widehat{\phi}_{c}(\mathbb{M} ; \boldsymbol{F}, c, \theta) \\ \text { 5. Constitutive heat flux vector } \mathbb{Q}=\partial_{\mathbb{G}} \widehat{\phi}_{t}(\mathbb{G} ; \boldsymbol{F}, c, \theta)\end{array}$

\subsubsection{Boundary conditions of the coupled problem}

The boundary $\partial \mathcal{B}_{0}$ of the body in the reference configuration is split into Dirichlet and Neumann boundaries for deformation $\varphi$, chemical potential $\mu$ and temperature $\theta$ fields

$$
\partial \mathcal{B}_{0}=\partial \mathcal{B}_{0}^{\varphi} \cup \partial \mathcal{B}_{0}^{t}, \quad \partial \mathcal{B}_{0}=\partial \mathcal{B}_{0}^{\mu} \cup \partial \mathcal{B}_{0}^{h}, \quad \partial \mathcal{B}_{0}=\partial \mathcal{B}_{0}^{\theta} \cup \partial \mathcal{B}_{0}^{q},
$$

such that

$$
\partial \mathcal{B}_{0}^{\varphi} \cap \partial \mathcal{B}_{0}^{t}=\emptyset, \quad \partial \mathcal{B}_{0}^{\mu} \cap \partial \mathcal{B}_{0}^{h}=\emptyset, \quad \partial \mathcal{B}_{0}^{\theta} \cap \partial \mathcal{B}_{0}^{q}=\emptyset .
$$

Given are the Dirichlet and Neumann boundary conditions for the deformation

$$
\boldsymbol{\varphi}=\overline{\boldsymbol{\varphi}}(\boldsymbol{X}, t) \quad \text { on } \partial \mathcal{B}_{0}^{\varphi} \quad \text { and } \quad \boldsymbol{P} \cdot \boldsymbol{N}=\overline{\boldsymbol{T}}(\boldsymbol{X}, t) \quad \text { on } \partial \mathcal{B}_{0}^{t},
$$

for the chemical potential

$$
\mu=\bar{\mu}(\boldsymbol{X}, t) \quad \text { on } \partial \mathcal{B}_{0}^{\mu} \quad \text { and } \quad \mathbb{H} \cdot \boldsymbol{N}=\bar{H}(\boldsymbol{X}, t) \quad \text { on } \partial \mathcal{B}_{0}^{h},
$$

and for the temperature

$$
\theta=\bar{\theta}(\boldsymbol{X}, t) \quad \text { on } \partial \mathcal{B}_{0}^{\theta} \quad \text { and } \quad \mathbb{Q} \cdot \boldsymbol{N}=\bar{Q}(\boldsymbol{X}, t) \quad \text { on } \partial \mathcal{B}_{0}^{q},
$$


with $\bar{\varphi}, \bar{\mu}$ and $\bar{\theta}$ being the prescribed deformation, chemical potential and temperature and with $\overline{\boldsymbol{T}}, \bar{H}$ and $\bar{Q}$ being the prescribed traction, species outflux and heat outflux. The governing equations of the coupled problem, which are used to derive the weak form, are summarized in the following box:

\begin{tabular}{|lc|}
\hline 1. Balance of linear momentum & $\operatorname{Div}[\boldsymbol{P}]+\boldsymbol{\gamma}=\mathbf{0}$ \\
2. Constitutive chemical potential & $\mu=\partial_{c} \widehat{\psi}$ \\
3. Balance of species content & $\dot{c}=-\operatorname{Div}[\mathbb{H}]$ \\
4. Temperature evolution equation $\mathbb{c} \dot{\theta}=-\operatorname{Div}[\mathbb{Q}]+\mathbb{H} \cdot \mathbb{M}+r+\mathcal{L}$ \\
\hline
\end{tabular}

Note that in the numerical treatment of our four-field setting, the constitutive equation for chemical potential $(48)_{2}$ has been treated as a global equation. The reason is that a three-field formulation in terms only of the primary field variables $\{\boldsymbol{\varphi}, \mu, \theta\}$ will lead to complications in evaluating the species flux at the boundary of the particles using the Butler-Volmer equation, see Sect. 3.3; thus, we consider the concentration $c$ as a primary (global) variable and regard the constitutive equation for chemical potential as an additional global equation.

\section{Model problem: electrode particles of $\mathrm{Li}$-ion batteries}

In what follows, we reduce the continuum formulation of Sect. 2 to the specific case of electrode particles in lithium-ion batteries. To this end, we present the specific choice of constitutive functions for the stored energy and the dissipation potentials. Then, we comment on the kinetics of electrochemical reaction at particle interface and finally, we discuss heat generation due to current exchange at the boundary of particles.

\subsection{Constitutive functions for the stored energy}

A multiplicative decomposition of the deformation gradient into an elastic part $\boldsymbol{F}_{e}$, a chemical part $\boldsymbol{F}_{c}$ and a thermal part $\boldsymbol{F}_{t}$ is considered:

$$
\boldsymbol{F}=\boldsymbol{F}_{e} \boldsymbol{F}_{c} \boldsymbol{F}_{t} \text { with } \boldsymbol{F}_{c}=\left(J_{c}\right)^{\frac{1}{3}} \boldsymbol{1} \text { and } \boldsymbol{F}_{t}=\left(J_{t}\right)^{\frac{1}{3}} \mathbf{1},
$$

where the chemical and thermal parts are volumetric deformations in terms of the chemical and thermal expansions

$$
J_{c}=1+\Omega\left(c-c_{0}\right) \quad \text { and } \quad J_{t}=\exp \left[3 \alpha\left(\theta-\theta_{0}\right)\right] .
$$

Here, $c_{0}$ and $\theta_{0}$ are the initial concentration and temperature, respectively. $\Omega$ is a swelling parameter and $\alpha$ is the coefficient of linear thermal expansion. The stored energy function is assumed to additively decompose into elastic, chemical and thermal contributions

$$
\widehat{\psi}(\boldsymbol{F}, c, \theta)=\widehat{\psi}_{e}(\boldsymbol{F}, c, \theta)+\widehat{\psi}_{c}(c)+\widehat{\psi}_{t}(\theta) .
$$

The elastic part $\widehat{\psi}_{e}$ of the stored energy function depends on the elastic part $\boldsymbol{F}_{e}$ of the deformation gradient and is of compressible Neo-Hookean type

$$
\widehat{\psi}_{e}=\frac{\gamma}{2}\left[\boldsymbol{F}_{e}: \boldsymbol{F}_{e}-3\right]+\frac{\gamma}{\zeta}\left[\left(\operatorname{det} \boldsymbol{F}_{e}\right)^{-\zeta}-1\right] .
$$

Here, $\gamma$ is the shear modulus and the parameter $\zeta>0$ depends on the Poisson ratio $v$ by the relationship $\zeta=\frac{2 v}{1-2 v}$. The purely chemical contribution to the stored energy function is given by

$$
\widehat{\psi}_{c}=A[c \ln c+(1-c) \ln (1-c)],
$$

where $A$ is a material constant. Finally, the purely thermal contribution to the stored energy function has the form

$$
\widehat{\psi}_{t}=-C\left[\theta \ln \left(\frac{\theta}{\theta_{0}}\right)-\left(\theta-\theta_{0}\right)\right]
$$

where the heat capacity $C$ is assumed to be constant. 
3.2 Constitutive functions for the dissipation potentials

A simple choice for the dissipation potential function discussed in Sect. 2.3.6 would be a quadratic function in terms of $\mathbb{M}$ and $\mathbb{G}$,

$$
\widehat{\phi}(\mathbb{M}, \mathbb{G} ; \boldsymbol{F}, c, \theta)=\frac{1}{2} \mathfrak{a}:(\mathbb{M} \otimes \mathbb{M})+\mathfrak{b}:(\mathbb{M} \otimes \mathbb{G})+\frac{1}{2} \mathfrak{c}:(\mathbb{G} \otimes \mathbb{G}),
$$

where $\mathfrak{a}=\mathfrak{a}^{T}$ and $\mathfrak{c}=\mathfrak{c}^{T}$, while the constraint (35) holds and the block $\mathfrak{a}$ is positive semi-definite $\mathfrak{a} \succeq 0$. Note that $\mathfrak{a}, \mathfrak{b}$ and $\mathfrak{c}$ generally depend on deformation, concentration and temperature. The choice of a quadratic dissipation potential function leads to a linear dependence of $\mathcal{J}$ on $\mathcal{I}$ in the form $\mathcal{J}=\mathfrak{H} \cdot \mathcal{I}$, where $\mathfrak{H}$ is a symmetric positive semi-definite tensor independent of $\mathcal{I}$. Using the representation (34) of $\mathfrak{H}$, we can write

$$
\mathfrak{H}=\left[\begin{array}{ll}
\mathfrak{a} & \mathfrak{b} \\
\mathfrak{b}^{T} & \mathfrak{c}
\end{array}\right] \stackrel{\overrightarrow{\mathcal{J}=\mathfrak{H} \cdot \mathcal{I}}}{=} \quad\left\{\begin{array}{l}
\mathbb{H}=\mathfrak{a} \cdot \mathbb{M}+\mathfrak{b} \cdot \mathbb{G} \\
\mathbb{Q}=\mathfrak{b}^{T} \cdot \mathbb{M}+\mathfrak{c} \cdot \mathbb{G}
\end{array}\right.
$$

The tensor $\mathfrak{b}$ couples species flow to temperature gradient and heat flow to the gradient of chemical potential. These couplings are called the Soret effect and the Dufour effect, respectively, see De Groot and Mazur [16] and Rahman and Saghir [41]. Note that the symmetry of $\mathfrak{H}$ is in line with Onsager's reciprocal relations, see De Groot and Mazur [16].

We choose the following specific form for the dissipation potential function (55)

$$
\widehat{\phi}(\mathbb{M}, \mathbb{G} ; \boldsymbol{F}, c)=c(1-c) \boldsymbol{C}^{-1}:\left[\frac{M}{2}(\mathbb{M} \otimes \mathbb{M})+L(\mathbb{M} \otimes \mathbb{G})+\frac{K}{2}(\mathbb{G} \otimes \mathbb{G})\right],
$$

where $M, L$ and $K$ are material parameters and $C$ is the right Cauchy-Green tensor. Positive semi-definiteness of $\mathfrak{H}$ imposes a constraint on the material parameters $M, L$ and $K$. Since $c \in[0,1]$ and the inverse of the right Cauchy-Green tensor $\boldsymbol{C}^{-1}$ is symmetric and positive definite, based on (35), the following constraints need to be fulfilled such that the second law of thermodynamics is not violated:

$$
M \geq 0 \text { and } K M-L^{2} \geq 0 .
$$

In Sect. 5.1, we will comment on the response of the coupled thermo-chemo-mechanical model using the above formulation.

Setting $L=0$ in (57) and thus, $\mathfrak{b}=\mathbf{0}$ in (55) and (56), brings us the decoupled setting discussed in Sect. 2.3.7, where the following chemical dissipation potential function characterizes the species flow

$$
\widehat{\phi}_{c}(\mathbb{M} ; \boldsymbol{F}, c)=\frac{M}{2} c(1-c) \boldsymbol{C}^{-1}:(\mathbb{M} \otimes \mathbb{M}),
$$

at a given state of deformation represented by the right Cauchy-Green tensor $\boldsymbol{C}$ and concentration $c$. Here, $M>0$ is identified as the species mobility, see Miehe et al. [36]. The thermal dissipation potential function governs the heat flow and has a similar structure to the chemical dissipation potential in (59). It is a convex function in $\mathbb{G}$

$$
\widehat{\phi}_{t}(\mathbb{G} ; \boldsymbol{F}, c)=\frac{K}{2} c(1-c) \boldsymbol{C}^{-1}:(\mathbb{G} \otimes \mathbb{G}),
$$

at a given state of deformation and concentration, see Miehe et al. [37]. Here, the parameter $K>0$ represents the thermal conductivity.

\subsection{Electrochemical reactions at the boundary of particles}

During discharging of the battery, lithium ions migrate from anode through the electrolyte to cathode and the electrons flow in the external circuit in the same direction, whereas during charging, lithium ions migrate from cathode through the electrolyte to anode. An external voltage is needed for the charging process. The 


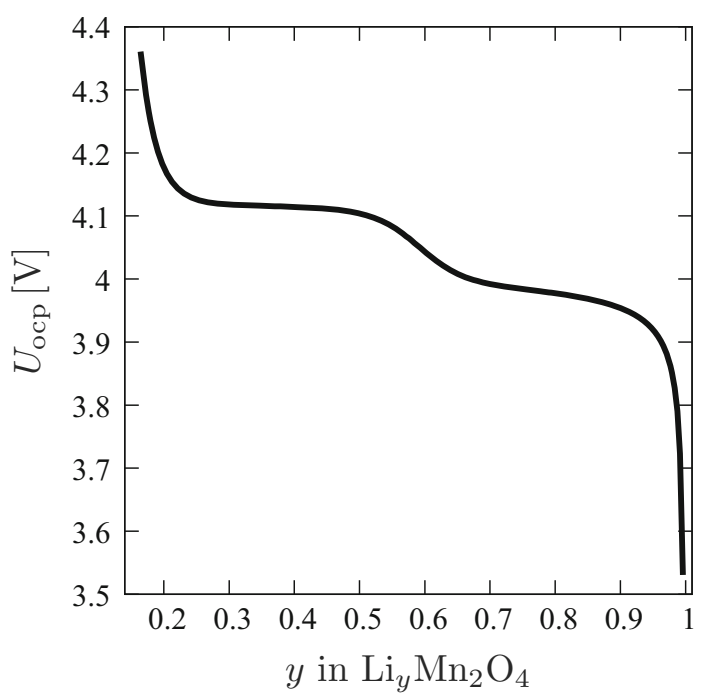

Fig. 2 The open-circuit potential of $\mathrm{LiMn}_{2} \mathrm{O}_{4}$ versus the state of charge based on the curve fitting, proposed in Doyle et al. [22], to the experimental measurements

reduction-oxidation reaction that takes place during charge/discharge cycles in the cathode of the lithium-ion battery under our consideration is

$$
\mathrm{LiMn}_{2} \mathrm{O}_{4} \underset{\text { discharge }}{\stackrel{\text { charge }}{\rightleftarrows}} \mathrm{Li}_{1-x} \mathrm{Mn}_{2} \mathrm{O}_{4}+x \mathrm{Li}^{+}+x \mathrm{e}^{-}
$$

Following the approach of Fuller et al. [24] and Newman and Thomas-Alyea [39], the rate of the electrochemical reaction is related to the surface overpotential $\eta_{s}$, which serves as the driving force of the reaction. Surface overpotential is the difference between the potential $V$ of the working electrode and the open-circuit potential $U_{\text {ocp }}$; thus, we have $\eta_{s}=V-U_{\text {ocp. }}$ The open-circuit potential $U_{\text {ocp }}$ is the potential of an electrode of the same material which is in equilibrium with the electrolyte. It is a function of the state of charge, which is expressed by the stoichiometric ratio $y=1-x$ of lithium in the electrode $\mathrm{Li}_{y} \mathrm{Mn}_{2} \mathrm{O}_{4}$. This can be considered as equivalent to the unitless concentration $c$ of lithium ions in the electrode. Following the work of Doyle et al. [22], the open-circuit potential of $\mathrm{LiMn}_{2} \mathrm{O}_{4}$ is given in terms of the state of charge by the curve of Fig. 2 . Additionally, similar to Zhang et al. [55] we can assume that, due to the small size of the particle, the potential within the particle is homogeneous and equal to the applied potential $U_{\text {applied }}$ under potentiodynamic stimulus. We can then summarize

$$
\eta_{s}=V(t)-U_{\mathrm{ocp}}(c) \quad \text { with } \quad V(t)=U_{\text {applied }} .
$$

The current density $i$ at the surface of the particle is related to the surface overpotential $\eta_{s}$ by the Butler-Volmer equation

$$
i=i_{0}\left\{\exp \left[\frac{(1-\beta) F}{R \theta} \eta_{s}\right]-\exp \left[\frac{-\beta F}{R \theta} \eta_{s}\right]\right\}
$$

Here, $i_{0}$ is the exchange current density, $\beta$ is the symmetry factor, $R$ is the gas constant and $F$ is Faraday's constant. The exchange current density $i_{0}$ is given by

$$
i_{0}=F k \tilde{c}_{\max }\left(\tilde{c}_{\mathrm{amb}}\right)^{(1-\beta)}(1-c)^{(1-\beta)} c^{\beta},
$$

where $\tilde{c}_{\mathrm{amb}}$ is the molar concentration of lithium ions in the electrolyte and $k$ is the reaction rate constant. Finally, the flux of lithium ions through the particle surface is given by

$$
\bar{H}=\frac{i}{F \tilde{c}_{\max }}=k\left(\tilde{c}_{\mathrm{amb}}\right)^{(1-\beta)}(1-c)^{(1-\beta)} c^{\beta}\left\{\exp \left[\frac{(1-\beta) F}{R \theta} \eta_{s}\right]-\exp \left[\frac{-\beta F}{R \theta} \eta_{s}\right]\right\} .
$$

We will assume that concentration of lithium ions in the electrolyte $\tilde{c}_{\mathrm{amb}}$ remains almost constant during the operation of the battery. Hence, we consider the multiplier $k\left(\tilde{c}_{\mathrm{amb}}\right)^{(1-\beta)}$ as a model parameter and denote it by $H^{\mathrm{b}}$ in the forthcoming sections. 


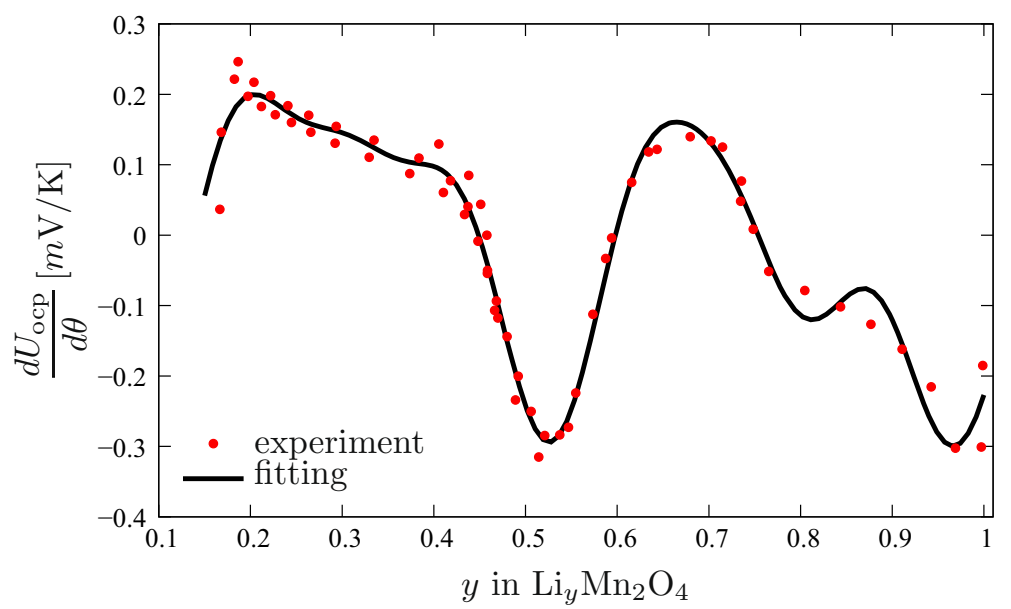

Fig. $3 \frac{d U_{\text {ocp }}}{d \theta}$ of $\mathrm{LiMn}_{2} \mathrm{O}_{4}$ plotted versus the state of charge. A curve fitting to the experimental measurements of Thomas et al. [46] has been performed using the sum of sines with 4 terms in MATLAB

\subsection{Heat generation due to current exchange}

Neglecting side reactions, we assume, similar to Masmoudi et al. [35], that heat generation within the particle can be evaluated based on the current exchange at the interface of the particle. Two contributions to the generated heat are considered, namely the irreversible resistive heating and the reversible entropic heating. The resistive heating is denoted by $R_{r}$ and is given in the form of the following integral over the surface of the particle

$$
R_{r}=\int_{\partial \mathcal{B}_{0}} i \eta_{s} \mathrm{~d} A
$$

where $i$ is the current density determined by the Butler-Volmer equation (63) and $\eta_{s}$ is the surface overpotential (62), see Sect. 3.3. The entropic heating $R_{e}$ is given by

$$
R_{e}=\int_{\partial \mathcal{B}_{0}} i \theta \frac{d U_{\mathrm{ocp}}}{d \theta} \mathrm{d} A
$$

where $\frac{d U_{\text {ocp }}}{d \theta}$ is a function of the state of charge. Similar to Zhang et al. [57], we use the experimental measurements of Thomas et al. [46] for evaluating $\frac{d U_{\text {ocp }}}{d \theta}$ of $\mathrm{LiMn}_{2} \mathrm{O}_{4}$ as a function of the state of charge. A curve fitting, shown in Fig. 3, to the experimental measurements has been performed using the sum of sines with 4 terms in MATLAB. This specific choice has been made to avoid the complexities of implementing a piecewise function in our numerical treatment. The fit is given by

$$
\begin{aligned}
\frac{d U_{\text {ocp }}}{d \theta}= & a_{1} \sin \left(b_{1} y+c_{1}\right)+a_{2} \sin \left(b_{2} y+c_{2}\right) \\
& +a_{3} \sin \left(b_{3} y+c_{3}\right)+a_{4} \sin \left(b_{4} y+c_{4}\right),
\end{aligned}
$$

where the state of charge $y$ is equal to the unitless concentration $c$ and the set of fitting parameters are

$$
\left\{\begin{array}{lll}
a_{1}=0.07256 & b_{1}=4.02 & c_{1}=1.08 \\
a_{2}=0.1061 & b_{2}=27.24 & c_{2}=-3.571 \\
a_{3}=0.1705 & b_{3}=15.04 & c_{3}=-2.828 \\
a_{4}=0.02812 & b_{4}=43.34 & c_{4}=0.8168
\end{array}\right.
$$

The overall heat generation due to current exchange would be the sum of (66) and (67)

$$
R=R_{e}+R_{r},
$$


which will be applied within the particle as a local heat source. To this end, we will use the following equation to evaluate the local heat source $r$ per unit reference volume

$$
R=\int_{\mathcal{B}_{0}} r \mathrm{~d} V
$$

We will assume a homogeneous distribution of the local heat source $r$ in $\mathcal{B}_{0}$. Thus, one can write

$$
r=\frac{R}{\left|\mathcal{B}_{0}\right|}
$$

where $\left|\mathcal{B}_{0}\right|$ denotes the total volume of $\mathcal{B}_{0}$.

\section{Time and space discretization}

\subsection{Time discretization}

Applying an implicit time integration scheme on the governing equations of the coupled problem (48), we end up with the set of time-discrete equations. To this end, we consider a time step $\left[t_{n}, t_{n+1}\right]$ and assume that all of the variables at time $t_{n}$ are known. All variables at time $t_{n}$ are shown with a subscript $n$. For the sake of simplicity, we skip the subscript $n+1$ for the variable under consideration at the current time $t_{n+1}$. Let $\tau=t_{n+1}-t_{n}$ denote the time step size. The time-discrete form of the balance of species content $(48)_{3}$ and the temperature evolution Eq. (48) 4 are given by

$$
\frac{c-c_{n}}{\tau}=-\operatorname{Div}[\mathbb{H}]
$$

and

$$
-\theta \partial_{\theta \theta}^{2} \widehat{\psi}\left[\frac{\theta-\theta_{n}}{\tau}\right]=-\operatorname{Div}[\mathbb{Q}]+\mathbb{H} \cdot \mathbb{M}+r_{n}+\theta \partial_{\boldsymbol{F} \theta}^{2} \widehat{\psi}:\left[\frac{\boldsymbol{F}-\boldsymbol{F}_{n}}{\tau}\right]+\theta \partial_{c \theta}^{2} \widehat{\psi}\left[\frac{c-c_{n}}{\tau}\right]
$$

Note that in (74), the heat source $r$ is frozen at its previous value $r_{n}$ at time $t_{n}$. This approximation simplifies the numerical treatment of the problem dramatically. We only need to evaluate the current heat source $r$ and save it as a history variable to be used in the next time step.

\subsection{Space discretization}

We construct the weak form of the problem using a standard Galerkin method. For this purpose, the timediscrete forms of the governing equations are multiplied by arbitrarily chosen test functions out of the admissible function spaces listed below:

$$
\begin{aligned}
& \delta \varphi \in W_{\varphi}^{0}:=\left\{\delta \varphi \mid \delta \varphi=\mathbf{0} \text { on } \partial \mathcal{B}_{0}^{\varphi}\right\}, \\
& \delta \mu \in W_{\mu}^{0}:=\left\{\delta \mu \mid \delta \mu=0 \text { on } \partial \mathcal{B}_{0}^{\mu}\right\}, \\
& \delta c \in W_{c}^{0}:=\left\{\delta c \mid \delta c=0 \text { on } \partial \mathcal{B}_{0}^{c}=\emptyset\right\}, \\
& \delta \theta \in W_{\theta}^{0}:=\left\{\delta \theta \mid \delta \theta=0 \text { on } \partial \mathcal{B}_{0}^{\theta}\right\}
\end{aligned}
$$


The weak forms of the governing equations are then obtained as

$$
\begin{aligned}
& \int_{\mathcal{B}_{0}}-\{\operatorname{Div}[\boldsymbol{P}]+\boldsymbol{\gamma}\} \cdot \delta \boldsymbol{\varphi} \mathrm{d} V \quad=0, \\
& \int_{\mathcal{B}_{0}}^{\mathcal{B}_{0}}-\left\{\mu-\partial_{c} \widehat{\psi}\right\} \delta c \mathrm{~d} V=0, \\
& \int_{\mathcal{B}_{0}}^{\mathcal{B}_{0}}-\left\{\left(c-c_{n}\right)+\tau \operatorname{Div}[H]\right\} \delta \mu \mathrm{d} V \quad=0, \\
& \int_{\mathcal{B}_{0}}\left\{\partial_{\theta \theta}^{2} \widehat{\psi}\left[\theta-\theta_{n}\right]+\partial_{\boldsymbol{F} \theta}^{2} \widehat{\psi}:\left[\boldsymbol{F}-\boldsymbol{F}_{n}\right]\right. \\
& \left.+\partial_{c \theta}^{2} \widehat{\psi}\left[c-c_{n}\right]-\frac{\tau}{\theta} \operatorname{Div}[\mathbb{Q}]+\frac{\tau}{\theta} \mathbb{H} \cdot \mathbb{M}+\frac{\tau}{\theta} r_{n}\right\} \delta \theta \mathrm{d} V=0,
\end{aligned}
$$

for all admissible test functions $\delta \boldsymbol{\varphi}, \delta \mu, \delta c$ and $\delta \theta$. Using Gauss's theorem, the Cauchy-like relationships (7), (10) and (13), and the Neumann boundary conditions $(45)_{2},(46)_{2}$ and $(47)_{2}$, the above equations can be rewritten as

$$
\begin{array}{ll}
\int_{\mathcal{B}_{0}}\{\boldsymbol{P}: \nabla \delta \boldsymbol{\varphi}-\boldsymbol{\gamma} \cdot \delta \boldsymbol{\varphi}\} \mathrm{d} V-\int_{\partial \mathcal{B}_{0}^{t}} \overline{\boldsymbol{T}} \cdot \delta \boldsymbol{\varphi} \mathrm{d} A & =0, \\
\int_{\mathcal{B}_{0}}^{\mathcal{B}_{0}}\left\{\partial_{c} \widehat{\psi}-\mu\right\} \delta c \mathrm{~d} V & =0, \\
\int_{\mathcal{B}_{0}}\left\{-\left(c-c_{n}\right) \delta \mu+\tau \mathbb{H} \cdot \nabla \delta \mu\right\} \mathrm{d} V-\int_{\partial \mathcal{B}_{0}^{h}} \tau \bar{H} \delta \mu \mathrm{d} A & =0, \\
\int_{\mathcal{B}_{0}}\left\{\partial_{\theta \theta}^{2} \widehat{\psi}\left[\theta-\theta_{n}\right]+\partial_{\boldsymbol{F} \theta}^{2} \widehat{\psi}:\left[\boldsymbol{F}-\boldsymbol{F}_{n}\right]+\partial_{c \theta}^{2} \widehat{\psi}\left[c-c_{n}\right]-\frac{\tau}{\theta^{2}} \mathbb{Q} \cdot \nabla \theta\right. & \\
\left.\left.+\frac{\tau}{\theta} \mathbb{H} \cdot \mathbb{M}+\frac{\tau}{\theta} r_{n}\right)\right\} \delta \theta \mathrm{d} V+\int_{\mathcal{B}_{0}} \frac{\tau}{\theta} \mathbb{Q} \cdot \nabla \delta \theta \mathrm{d} V-\int_{\partial \mathcal{B}_{0}^{q}} \frac{\tau}{\theta} \bar{Q} \delta \theta \mathrm{d} A & =0 .
\end{array}
$$

Note that in Eq. (77) ${ }_{3}$, the species flux at the boundary of the particle $\bar{H}$ is evaluated using the Butler-Volmer equation (65). For this reason, in addition to discretization of the referential volume $\mathcal{B}_{0}$, a discretization of the Neumann boundary $\partial \mathcal{B}_{0}^{h}$ using surface elements is required. Let us consider a finite element discretization of the bulk of $\mathcal{B}_{0}$ and the Neumann boundaries $\partial \mathcal{B}_{0}^{t}, \partial \mathcal{B}_{0}^{h}$ and $\partial \mathcal{B}_{0}^{q}$ as follows:

$$
\mathcal{B}_{0}=\bigcup_{e=1}^{N} \mathcal{B}_{0}^{e} \quad ; \quad \partial \mathcal{B}_{0}^{t}=\bigcup_{s=1}^{N^{t}} \partial \mathcal{B}_{0}^{t, s} \quad ; \quad \partial \mathcal{B}_{0}^{h}=\bigcup_{s=1}^{N^{h}} \partial \mathcal{B}_{0}^{h, s} \quad ; \quad \partial \mathcal{B}_{0}^{q}=\bigcup_{s=1}^{N^{q}} \partial \mathcal{B}_{0}^{q, s} .
$$

Here, $N$ is the number of the bulk finite elements, whereas $N^{t}, N^{h}$ and $N^{q}$ are the numbers of the surface finite elements used for the discretization of traction, species flux and heat flux boundaries. The following set of approximations have been used for the primary variables and the corresponding gradients within the bulk elements $\mathcal{B}_{0}^{e} \subset \mathcal{B}_{0}$

$$
\begin{aligned}
& \varphi^{h}=N_{\varphi}^{e} d_{\varphi} \\
& c^{h}=\boldsymbol{N}_{c}^{e} \boldsymbol{d}_{c} \\
& \mu^{h}=\boldsymbol{N}_{\mu}^{e} \boldsymbol{d}_{\mu} \\
& \theta^{h}=\boldsymbol{N}_{\theta}^{e} \boldsymbol{d}_{\theta} \\
& \text { and } \quad \nabla \mu^{h}=\boldsymbol{B}_{\mu}^{e} \boldsymbol{d}_{\mu} \\
& \nabla \theta^{h}=\boldsymbol{B}_{\theta}^{e} \boldsymbol{d}_{\theta}
\end{aligned}
$$

Here, $\boldsymbol{d}_{\varphi}, \boldsymbol{d}_{c}, \boldsymbol{d}_{\mu}$ and $\boldsymbol{d}_{\theta}$ are the vectors of nodal displacements, concentrations, chemical potentials and temperatures. The matrices $N_{\varphi}^{e}, N_{c}^{e}, N_{\mu}^{e}$ and $N_{\theta}^{e}$ include the shape functions used for the approximation of the fields within the elements, whereas $\boldsymbol{B}_{\varphi}^{e}, \boldsymbol{B}_{\mu}^{e}$ and $\boldsymbol{B}_{\theta}^{e}$ include the derivatives of the shape functions. The same 
set of shape functions has been used for the approximation of the virtual displacements $\delta \varphi$, concentrations $\delta c$, chemical potentials $\delta \mu$ and temperatures $\delta \theta$. The following approximations of the primary fields within the surface elements $\partial \mathcal{B}_{0}^{t, s} \subset \partial \mathcal{B}_{0}^{t}, \partial \mathcal{B}_{0}^{h, s} \subset \partial \mathcal{B}_{0}^{h}$ and $\partial \mathcal{B}_{0}^{q, s} \subset \partial \mathcal{B}_{0}^{q}$ are used:

$$
\begin{aligned}
\varphi^{h} & =\boldsymbol{N}_{\varphi}^{s} \boldsymbol{d}_{\varphi}, \\
c^{h} & =\boldsymbol{N}_{c}^{s} \boldsymbol{d}_{c}, \\
\mu^{h} & =\boldsymbol{N}_{\mu}^{s} \boldsymbol{d}_{\mu}, \\
\theta^{h} & =\boldsymbol{N}_{\theta}^{s} \boldsymbol{d}_{\theta} .
\end{aligned}
$$

Note that these shape functions should be compatible with the shape functions used for the bulk elements. Introducing the above approximations into the weak form (77) leads to the residual expressions

$$
\begin{aligned}
& \boldsymbol{R}_{\varphi}\left(\boldsymbol{d}_{\varphi}, \boldsymbol{d}_{c}, \boldsymbol{d}_{\mu}, \boldsymbol{d}_{\theta}\right)=\underset{e=1}{\mathrm{~A}} \int_{\mathcal{B}_{0}^{e}}\left\{\boldsymbol{B}_{\varphi}^{e T} \boldsymbol{P}-\boldsymbol{N}_{\varphi}^{e T} \boldsymbol{\gamma}\right\} \mathrm{d} V-\underset{s=1}{\mathrm{~A}^{t}} \int_{\partial \mathcal{B}_{0}^{t, s}} \boldsymbol{N}_{\varphi}^{s^{T}} \overline{\boldsymbol{T}} \mathrm{d} A=\mathbf{0}, \\
& \boldsymbol{R}_{c}\left(\boldsymbol{d}_{\varphi}, \boldsymbol{d}_{c}, \boldsymbol{d}_{\mu}, \boldsymbol{d}_{\theta}\right)=\underset{e=1}{\mathrm{~A}} \int_{\mathcal{B}_{0}^{e}} \boldsymbol{N}_{c}^{e T}\left(\partial_{c} \widehat{\psi}-\mu\right) \mathrm{d} V=\mathbf{0} \\
& \boldsymbol{R}_{\mu}\left(\boldsymbol{d}_{\varphi}, \boldsymbol{d}_{c}, \boldsymbol{d}_{\mu}, \boldsymbol{d}_{\theta}\right)=\mathrm{A}_{e=1}^{N} \int_{\mathcal{B}_{0}^{e}}\left\{\tau \boldsymbol{B}_{\mu}^{e T} \mathbb{H}-\boldsymbol{N}_{\mu}^{e T}\left(c-c_{n}\right)\right\} \mathrm{d} V-\AA_{s=1}^{N^{h}} \int_{\partial \mathcal{B}_{0}^{h, s}} \tau N_{\mu}^{s} T \bar{H} \mathrm{~d} A=\mathbf{0}, \\
& \boldsymbol{R}_{\theta}\left(\boldsymbol{d}_{\varphi}, \boldsymbol{d}_{c}, \boldsymbol{d}_{\mu}, \boldsymbol{d}_{\theta}\right)=\mathrm{A}_{e=1}^{N} \int_{\mathcal{B}_{0}^{e}} \boldsymbol{N}_{\theta}^{e T}\left\{\partial_{\theta \theta}^{2} \widehat{\psi}\left[\theta-\theta_{n}\right]+\partial_{\boldsymbol{F} \theta}^{2} \widehat{\psi}:\left[\boldsymbol{F}-\boldsymbol{F}_{n}\right]+\partial_{c \theta}^{2} \widehat{\psi}\left[c-c_{n}\right]\right. \\
& \left.-\frac{\tau}{\theta^{2}} \mathbb{Q} \cdot \nabla \theta+\frac{\tau}{\theta} \mathbb{H} \cdot \mathbb{M}+\frac{\tau}{\theta} r_{n}\right\} \mathrm{d} V \\
& +\underset{e=1}{\mathrm{~A}} \int_{\mathcal{B}_{0}^{e}} \frac{\tau}{\theta} \boldsymbol{B}_{\theta}^{e T} \mathbb{Q} \mathrm{d} V-\stackrel{N}{N}_{s=1} \int_{\partial \mathcal{B}_{0}^{q, s}} \frac{\tau}{\theta} \boldsymbol{N}_{\theta}^{s T} \bar{Q} \mathrm{~d} A=\mathbf{0} .
\end{aligned}
$$

The above coupled system has to be solved for the nodal displacements $\boldsymbol{d}_{\varphi}$, concentrations $\boldsymbol{d}_{c}$, chemical potentials $\boldsymbol{d}_{\mu}$ and temperatures $\boldsymbol{d}_{\theta}$. Let us introduce the following shorthand notation for the global vectors of residuals and generalized nodal displacements

$$
\boldsymbol{R}:=\left[\boldsymbol{R}_{\varphi}, \boldsymbol{R}_{c}, \boldsymbol{R}_{\mu}, \boldsymbol{R}_{\theta}\right]^{T} \text { and } \boldsymbol{d}:=\left[\boldsymbol{d}_{\varphi}, \boldsymbol{d}_{c}, \boldsymbol{d}_{\mu}, \boldsymbol{d}_{\theta}\right]^{T} .
$$

The problem under consideration is then to find $\boldsymbol{d}$ such that $\boldsymbol{R}(\boldsymbol{d})=\mathbf{0}$. A standard Newton-Raphson scheme can be used to solve this system of nonlinear equations. The vector of generalized nodal displacements is updated by

$$
d \Leftarrow d-[K]^{-1} R
$$

until a convergence criterion is met, e.g., $|\boldsymbol{R}|<$ tol. Here, $\boldsymbol{K}$ is the tangent of the Newton-Raphson scheme.

\section{Evaluation of the model response}

In what follows, we will show the results of a few numerical simulations obtained using the model proposed in the previous sections. In Sect. 5.1, we will comment on the results obtained by the coupled formulation of the dissipation potential, which was discussed in Sect. 2.3.6. This academic study does not directly apply to modeling of electrode particles in Li-ion batteries. The latter topic will be treated later in Sects. 5.2 and 5.3, where we will discuss the response of two cathode particles with different geometries under a potentio-dynamic loading. 


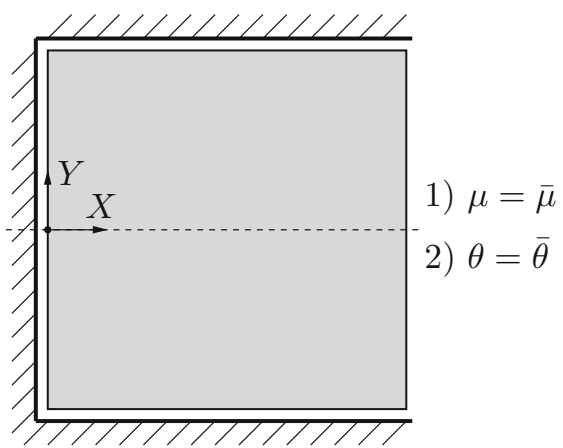

(a)

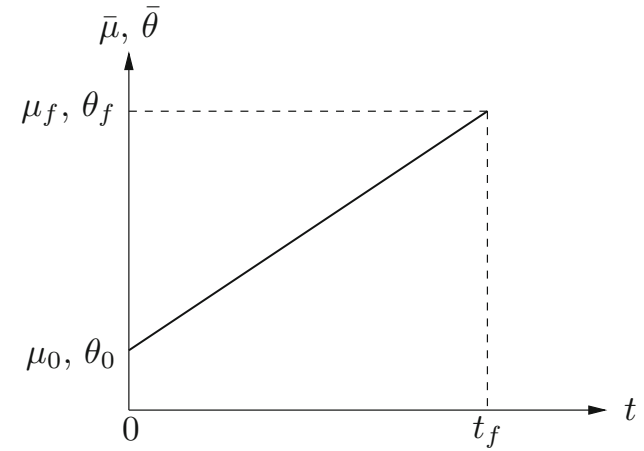

(b)

Fig. 4 Boundary conditions and the loading scenarios. On the top, bottom and left sides of the square block, zero heat and species fluxes are applied. On the right side of the block two loading cases are considered: (1) linear chemical potential increase from $\mu_{0}=0 \mathrm{GPa}$ to $\mu_{f}=5 \mathrm{GPa}$ and (2) linear temperature increase from $\theta_{0}=300 \mathrm{~K}$ to $\theta_{f}=600 \mathrm{~K}$

Table 1 Material and model parameters

\begin{tabular}{lllll}
\hline No. & Parameter & Name & Value & Unit \\
\hline 1. & $\gamma$ & Shear modulus & 3.0 & $\mathrm{GPa}$ \\
2. & $v$ & Poisson's ratio & 0.3 & - \\
3. & $\Omega$ & Swelling parameter & 0.1 & - \\
4. & $A$ & Chemical energy parameter & 1.0 & $\mathrm{mN} / \mu \mathrm{m}^{2}$ \\
5. & $M$ & Mobility parameter & 0.1 & $\mu \mathrm{m}^{4} /\left(\mathrm{mN}^{\mathrm{s}}\right)$ \\
6. & $\mathrm{~s}_{0}$ & Initial concentration & 0.5 & $1 / \mathrm{K}$ \\
7. & $\theta_{0}$ & Linear thermal expansion & $10^{-4}$ & $\mathrm{~K}$ \\
8. & $C$ & Initial temperature & 300 & $\mathrm{mN} /\left(\mu \mathrm{m}^{2} \cdot \mathrm{K}\right)$ \\
9. & $K$ & Heat capacity & $10^{-2}$ & $\mathrm{mN} / \mathrm{s}$ \\
10. & & Thermal conductivity & 10.0 & \\
\hline
\end{tabular}

\subsection{Model response of the fully coupled formulation}

To evaluate the model response of the fully coupled formulation discussed in Sect. 2.3.6, we consider two thermo-chemo-mechanical loading scenarios on a square block with the side length of $5 \mu \mathrm{m}$. The block is mechanically restricted such that it can only expand in horizontal direction, see Fig. 4a. Plane strain conditions are assumed. The material parameters used for this study are listed in Table 1 and do not represent any real material. For the discretization of the sample, elements with bi-linear shape functions for all of the unknown fields are used. Since no species flux boundary conditions are considered, there is no need to use surface elements at the boundaries.

In the first numerical experiment, a linearly increasing chemical potential $\mu=\bar{\mu}$ is applied on the right side of the block. See Fig. $4 \mathrm{~b}$ for the loading function. No species flux is assumed to take place on all of the other sides. Furthermore, no heat flux through the boundaries is permitted. The problem is indeed a one-dimensional problem and the primary fields vary only in $X$ direction. For three different values of the coupling parameter $L=\{0.0,0.5,-0.5\} \mu \mathrm{m}^{2} / \mathrm{s}$, introduced in Sect. 3.2, the values of species concentration $c$, chemical potential $\mu$ and temperature $\theta$ are plotted on the mid-line of the block in Fig. 5 at the final stage of loading $t_{f}=100 \mathrm{~s}$. Let us consider the case when $L=0.0$, shown in the graphs by solid black lines. Since chemical potential is increased on the right side of the block, its gradient points in the $X$ direction. Thus, $\mathbb{M}$ points in the negative $X$ direction, which by (56) creates a flux vector $\mathbb{H}$ also in the negative $X$ direction. This leads to an increase of the concentration field within the block, which can be observed in Fig. 5a. Furthermore, by the temperature evolution Eq. (48) 4 , we can expect an increase of the temperature within the block, observed in Fig. 5c. For a positive $L$, an additional term in the negative $X$ direction will be added to the heat flux vector $\mathbb{Q}$ in (56). This term will lead to a temperature decrease on the right side of the block shown in the temperature graph. The opposite effect takes place for a negative $L$.

In the second numerical experiment, we apply a linear temperature rise $\theta=\bar{\theta}$ on the right side of the square, see Fig. 4. No species flux is permitted at the boundaries. Heat transfer is also prevented on the top, bottom and 

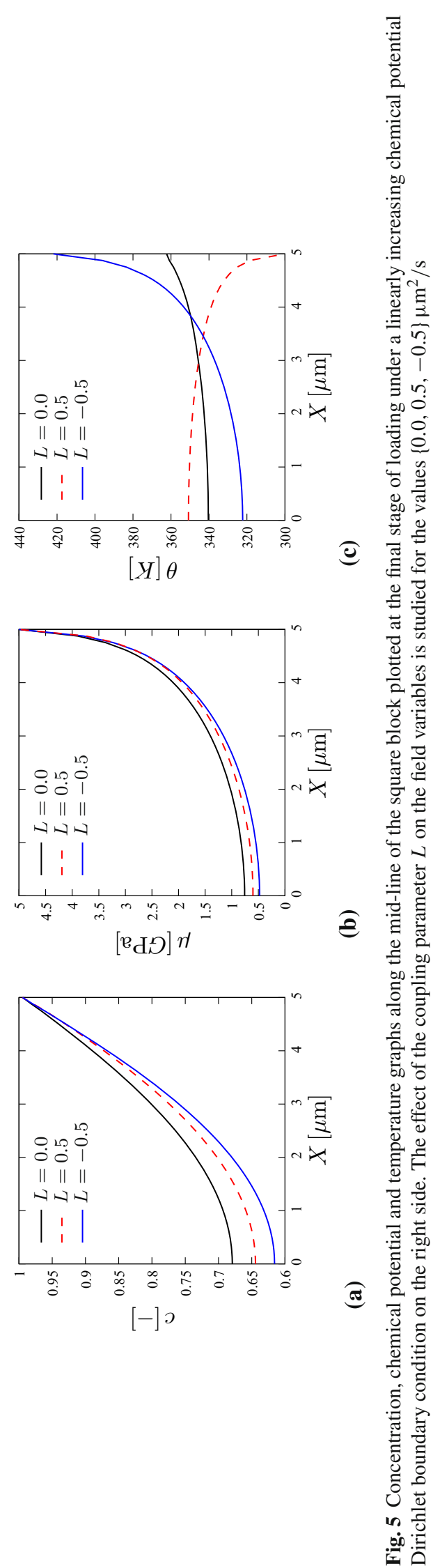

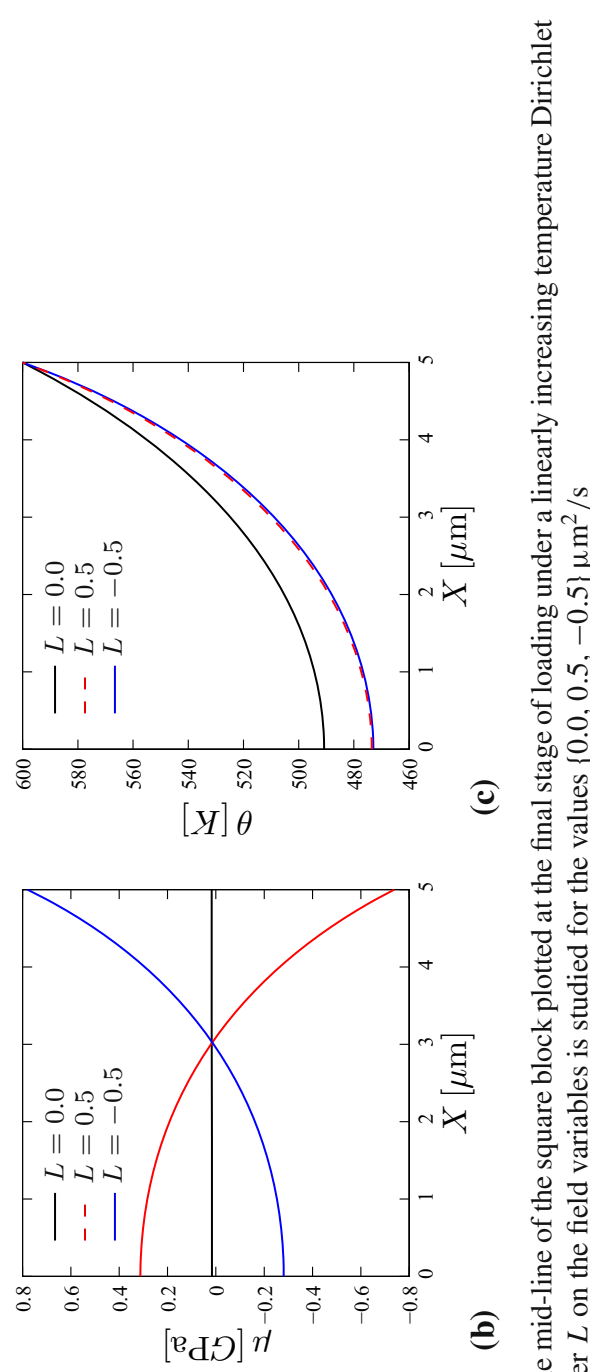

을

奇

몽

을

눙

$\frac{2}{3} \cdot \frac{2}{5}$

을

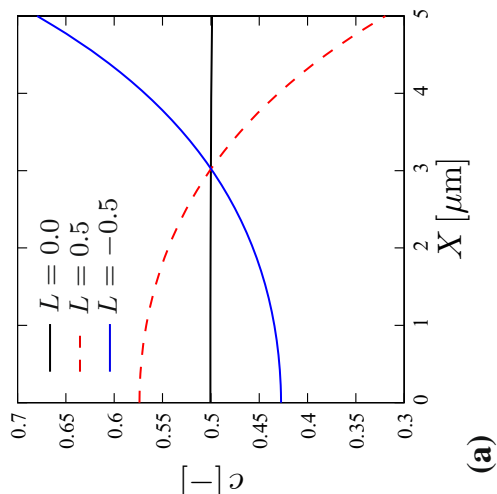

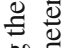




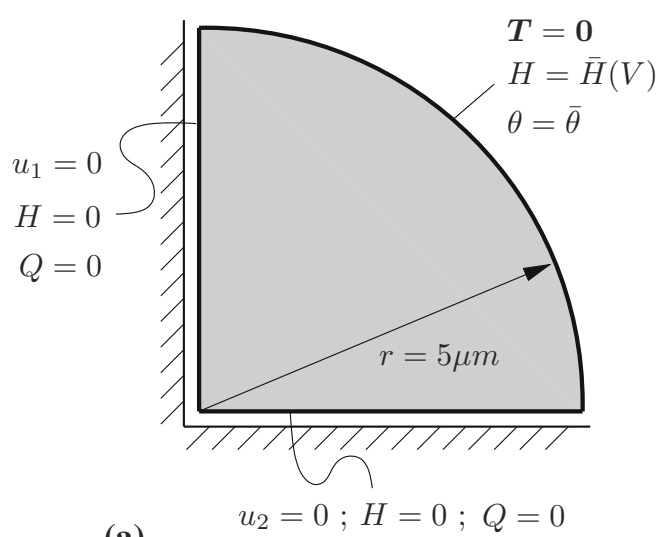

(a)

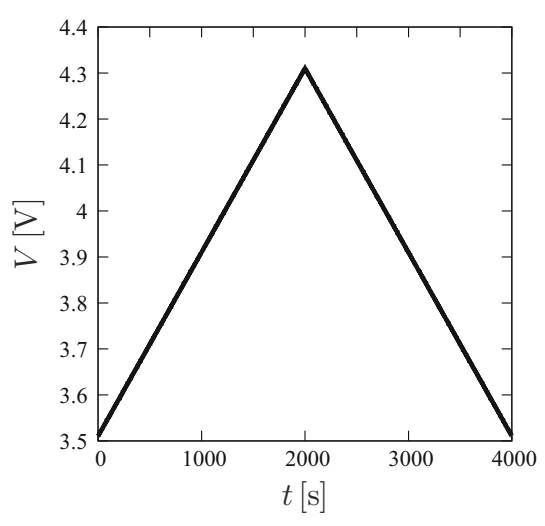

(b)

Fig. 7 a Boundary conditions applied on the circular particle. Due to symmetry, only one quarter of the particle is discretized. b The electric potential profile used by Zhang et al. [57] is applied on the particle. This induces a species flux through the boundary and simulates a charging-discharging cycle

left sides of the block. For the above-mentioned values of the coupling parameter $L$, the species concentration $c$, chemical potential $\mu$ and temperature $\theta$ plots on the mid-line of the block are given in Fig. 6 at the final stage of loading $t_{f}=100 \mathrm{~s}$. Even though the temperature graphs of Fig. $6 \mathrm{c}$ seem identical for $L=0.5 \mu \mathrm{m}^{2} / \mathrm{s}$ and $L=-0.5 \mu \mathrm{m}^{2} / \mathrm{s}$, the corresponding concentration graphs show a totally different pattern. For a positive $L$, an additional term in negative $X$ direction will be added to the species flux vector $\mathbb{H}$ in (56). This term will lead to a concentration decrease on the right side of the block and consequently, to a concentration rise on the left side observed in Fig. 6a. The opposite effect is observed for a negative $L$.

\subsection{A circular disk under potentio-dynamic loading}

We evaluate the model response by considering a circular $\mathrm{LiMn}_{2} \mathrm{O}_{4}$ cathode particle of radius $r=5 \mu \mathrm{m}$. The exterior of the particle is assumed to be free of traction. Additionally, it is assumed that the temperature at the boundary of the particle remains fixed since it is in constant contact with the electrolyte. We assume that the temperature of the electrolyte does not change. Due to symmetry, only a quarter of the particle is discretized by bi-linear bulk elements and linear surface elements. See Fig. 7a for the geometry of the particle and the applied boundary conditions. Plane strain conditions are considered in the simulation. A species flux through the boundary is induced by an electric potential applied on the particle. The applied electric potential follows the sweeping profile given in Zhang et al. [57], which simulates a charging-discharging cycle. See Fig. 7b for the applied electric potential. The initial values of the concentration and temperature are chosen to be $c_{0}=0.996$ and $\theta_{0}=298.15 \mathrm{~K}=25^{\circ} \mathrm{C}$ (room temperature), respectively. The material parameters given in Table 2 are chosen as realistically as possible under the assumptions of our modeling framework. The coupling parameter $L$ is set to zero.

The governing equations of the problem (48) are solved using the finite element method to evaluate the four unknown fields shown in Fig. 1. Flux of species $H$ induced by the applied potential is shown in Fig. 8a. In order to compare this result with the flux profile given in Zhang et al. [57], the computed values of $H$ are multiplied by the maximum molar concentration $\tilde{c}_{\max }=2.37 \times 10^{4} \mathrm{~mol} / \mathrm{m}^{3}$. Figure $8 \mathrm{~b}$ shows a comparison of the fluxes obtained by the simulation with the flux profile of Zhang et al. [57]. A relatively good agreement can be observed between the two results. Li-ion concentration versus time is shown in Fig. 9a at the center and on the surface of the particle. Due to the small size of the particle, variations of concentration within the particle are relatively small. This can be observed better in Fig. 9b, which shows the variations of Li-ion concentration within the particle in the radial direction. At some time instances, there are very small concentration gradients within the particle, while at some other instances, concentration of Li-ions can not be considered as homogeneous. The generated resistive and entropic heat at the particle interface are plotted in Fig. 10 in the first half of the loading cycle. As it can be observed in the figure, the entropic heat is more pronounced compared to resistive heat. The temperature evolution equation $(48)_{4}$ is solved by treating the overall generated heat $R_{e}+R_{r}$ as a homogeneously distributed source of heat inside the particle, determined by Eq. (72), and using the time 
Table 2 Material and model parameters

\begin{tabular}{llllll}
\hline No. & Parameter & Name & Value & Unit & Reference \\
\hline 1. & $\gamma$ & Shear modulus & 3.85 & $\mathrm{GPa}$ & Calculated based on [56] \\
2. & $\nu$ & Poisson's ratio & 0.3 & - & From [56] \\
3. & $\Omega$ & Swelling parameter & $8.29 \times 10^{-2}$ & - & Calculated based on [57] \\
4. & $A$ & Chemical energy parameter & $2.94 \times 10^{-2}$ & $\mathrm{mN} / \mu \mathrm{m}^{2}$ & Estimated based on [35] \\
5. & $M$ & Mobility parameter & 1.88 & $\mu \mathrm{m}^{4} /(\mathrm{mN} \cdot \mathrm{s})$ & Calculated based on [57] \\
6. & $c 0$ & Initial concentration & 0.996 & - & From [57] \\
7. & $\alpha$ & Linear thermal expansion & $10^{-5}$ & $1 / \mathrm{K}$ & Estimated based on [40] \\
8. & $\theta_{0}$ & Initial temperature & 298.15 & $\mathrm{~K}$ & - \\
9. & $C$ & Heat capacity & $1.05 \times 10^{-3}$ & $\mathrm{mN} /\left(\mu \mathrm{m}^{2} \cdot \mathrm{K}\right)$ & Calculated based on [2] \\
10. & $K$ & Thermal conductivity & $1.49 \times 10^{6}$ & $\mathrm{mN} / \mathrm{s}$ & Calculated based on [2] \\
11. & $\beta$ & Symmetry factor & 0.5 & - & From [57] \\
12. & $H^{b}$ & Flux parameter & $6.01 \times 10^{-2}$ & $\mu \mathrm{m} / \mathrm{s}$ & Calculated based on [55] \\
\hline
\end{tabular}
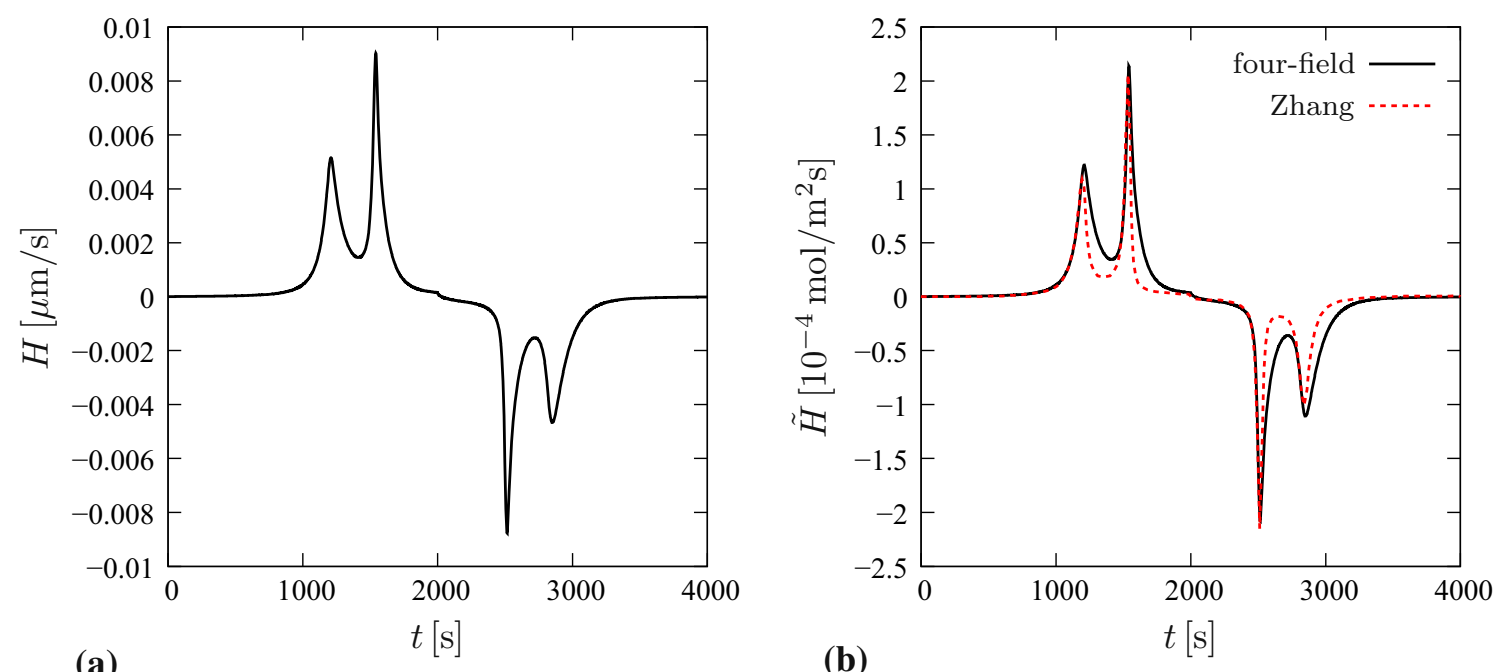

Fig. 8 a Species flux through the boundary of the particle plotted versus time. $H$ denotes the flux of the unitless species concentration $c$. b The flux shown on the left is multiplied by $\tilde{c}_{\max }$ to obtain the molar species flux $\tilde{H}$. It is then compared to the results obtained of Zhang et al. [57]

discretization approach discussed in Sect. 4.1. It turns out that the heat generated due to current exchange at the boundary of the particle as well as the heat generated due to diffusion and deformation do not have a major effect on the temperature inside the particle. Temperature within the particle remains almost constant and equal to the temperature of the surrounding environment. Hence, the dominant factor that determines the temperature inside the particle is the surrounding temperature. This observation can be attributed to the small size of the particle and hence, to the fast dissipation of the generated heat to the surroundings of the particle.

If instead of the Dirichlet temperature boundary condition at the boundary of the particle, shown in Fig. 7 , a Neumann boundary condition $Q=0$ for heat flux was considered, the temperature within the particle would increase dramatically. See Fig. 11 for the results of the simulation with zero flux boundary condition. This temperature rise is due to deformation, diffusion and heat generation caused by electric current exchange. These results underline once more the significance of heat dissipation to the surrounding environment without which, temperature rise is extreme.

\subsection{A two-dimensional particle geometry under potentio-dynamic loading}

In a second study, we consider a realistic two-dimensional geometry of a $\mathrm{LiMn}_{2} \mathrm{O}_{4}$ cathode particle shown in Fig. 12. This geometry is a two-dimensional reduction of the scanning electron microscope image provided in Bohn [5]. Similar boundary conditions to the previous example are assumed on the exterior of the particle, 


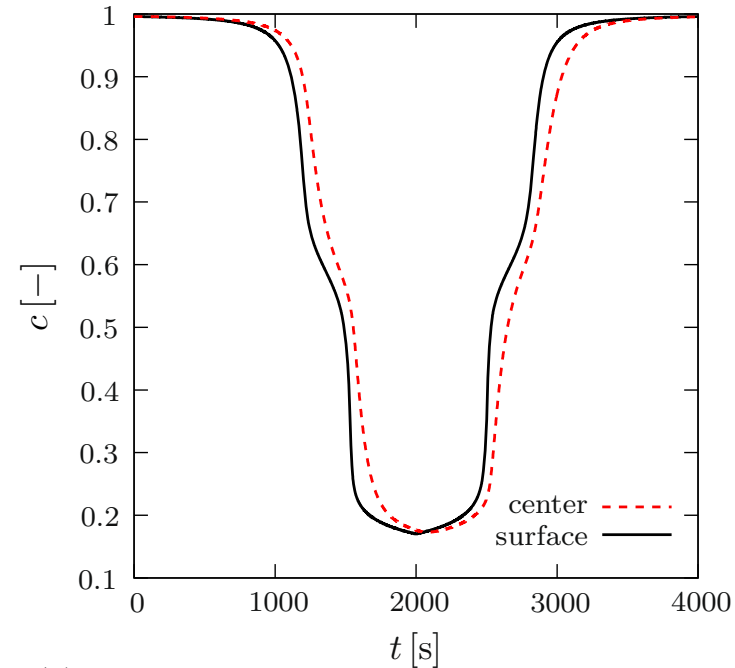

(a)

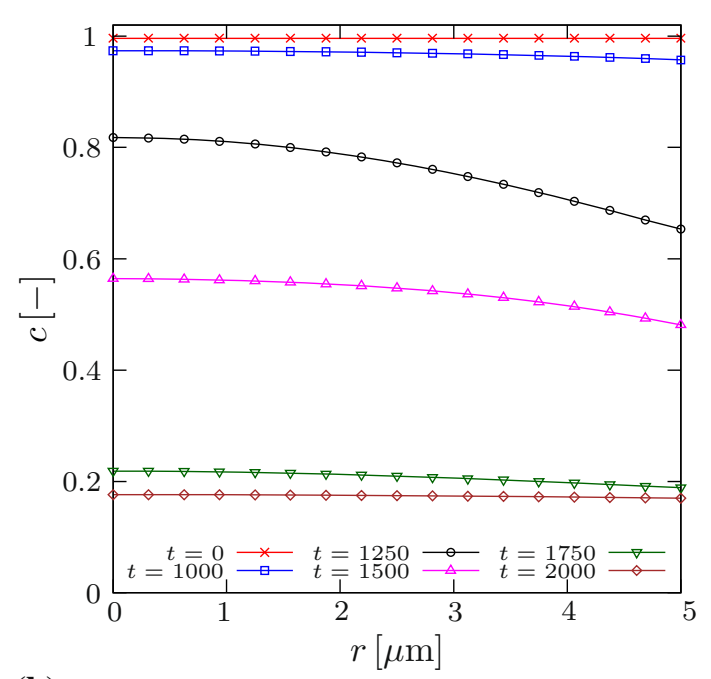

(b)

Fig. 9 a Species concentration plotted versus time at the center (red) and surface (black) of the particle. b Concentration profile in the radial direction plotted at different time instances during the first half of loading

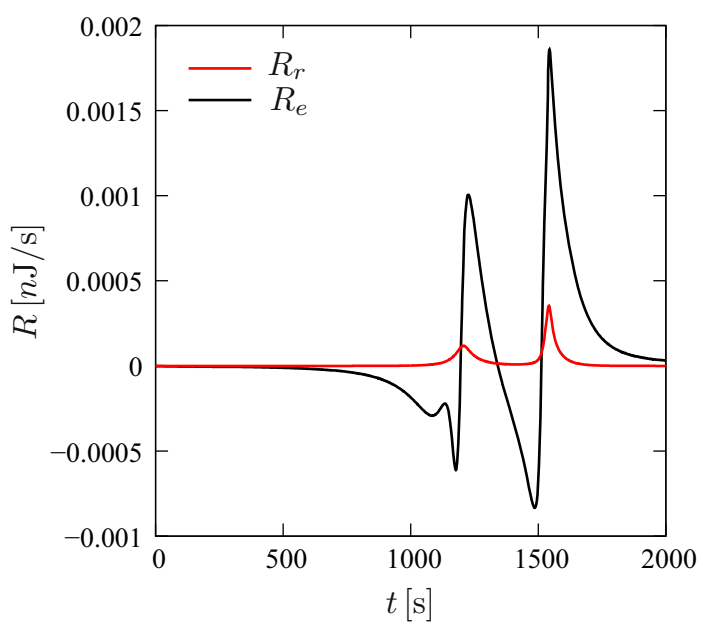

Fig. 10 Resistive heating $R_{r}$ and entropic heating $R_{e}$, given in Eqs. (66) and (67), are plotted versus time in the first half of the loading cycle. Integration over the entire surface of the particle is carried out with the help of the line finite elements. The overall heating $R_{e}+R_{r}$ is applied as a homogeneously distributed heat source inside the particle determined by Eq. (72)

i.e., the boundary of the particle is traction-free, the surrounding temperature is constant and a species flux on the boundary is induced by applying the electric potential profile shown in Fig. 7b. The initial conditions for concentration and temperature inside the particle are $c_{0}=0.996$ and $\theta_{0}=298.15 \mathrm{~K}$ (room temperature), respectively. The coupled problem is solved using the finite element method. The geometry has been discretized using 598 bulk elements and 100 surface elements. The material parameters of Table 2 are used for this problem. The coupling parameter $L$ is assumed to be equal to zero. Figure 13 shows the contour plots of the species concentration at different time instances in the first half of the charging-discharging cycle and also at the final time $t=4000 \mathrm{~s}$. As it can be observed in the figure, concentration of $\mathrm{Li}$-ions decreases during the first half of the loading, leading to a decrease in the volume of the particle. The initial volume of particle is recovered at the end of the loading cycle. The temperature rise within the particle is very small and can be ignored as far as the ambient temperature does not change. Once more, the ambient temperature turns out to govern the temperature evolution within the particle. This can be attributed to the small size of particle and fast dissipation of the produced heat to surrounding environment. 


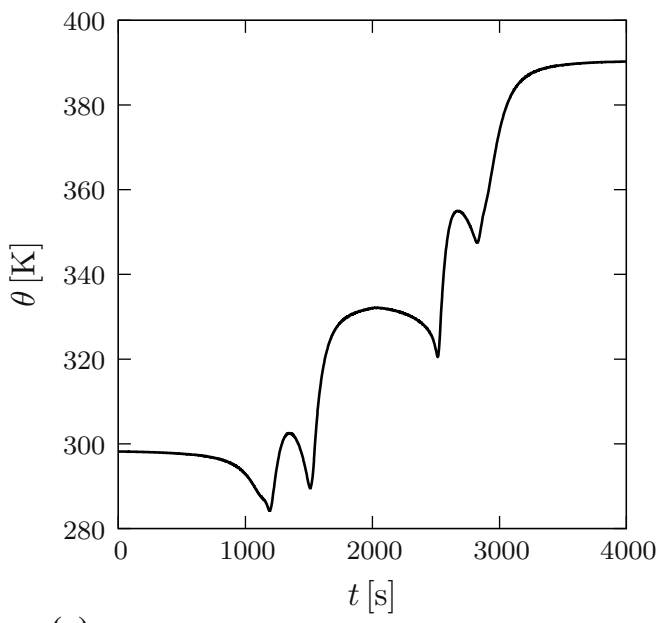

(a)

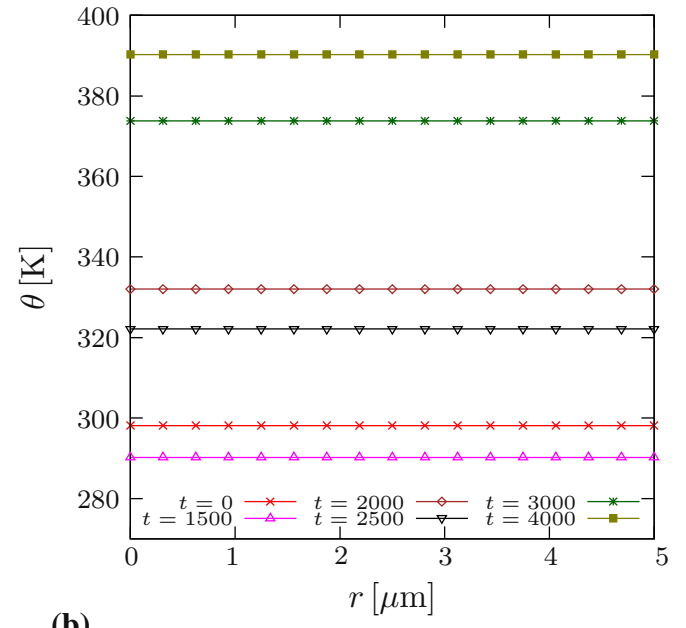

(b)

Fig. 11 Temperature plots for a zero Neumann boundary condition $\bar{Q}=0$. a Temperature at the center of the particle plotted versus time. b Temperature profile in the radial direction plotted at different time instances during the loading

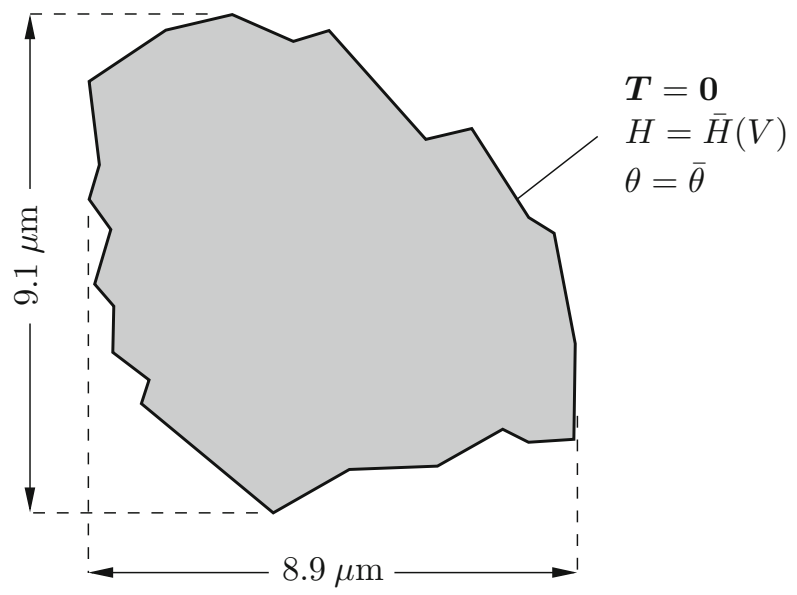

Fig. 12 Geometry and boundary conditions of the $\mathrm{LiMn}_{2} \mathrm{O}_{4}$ cathode particle. The electric potential profile shown in Fig. $7 \mathrm{~b}$ induces a species flux at the boundary. The ambient temperature is constant and no traction is present on the boundary

\section{Summary}

In this work, we presented a four-field formulation of coupled thermo-chemo-mechanical problems with application to the simulation of cathode particles in lithium-ion batteries. First, the governing equations of the coupled problem were discussed in a general framework. An alternative way of satisfying the second law of thermodynamics using Onsager's symmetric positive-definite matrix of coefficients was explained. This formulation enables us to take the Soret and Dufour effects into account. Subsequently, the coupled formulation was applied to the simulation of electrode particles in lithium-ion batteries. The generated heat due to current exchange at the boundary of the particle was considered as a volume heat source within the particle. Thereafter, time and space discretization of the problem were discussed. The response of the model was evaluated under a potentio-dynamic loading applied on different particle geometries. Temperature evolution within the particle was found to be very minor. The ambient temperature was found to be the most important factor governing the temperature distribution within the particle. 


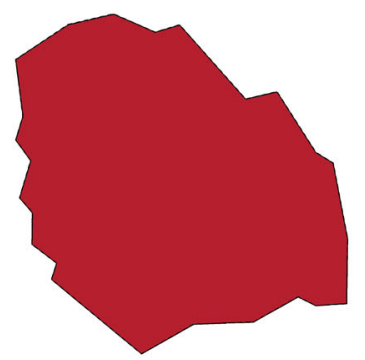

$t=0 \mathrm{~s}$

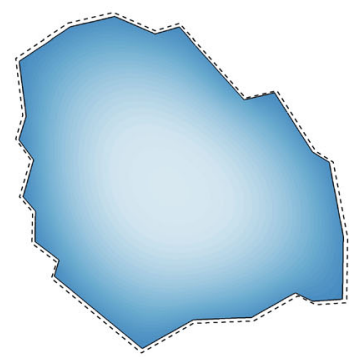

$t=1550 \mathrm{~s}$

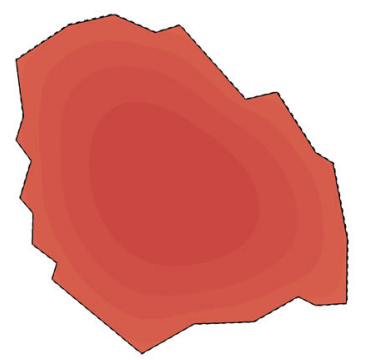

$t=1100 \mathrm{~s}$

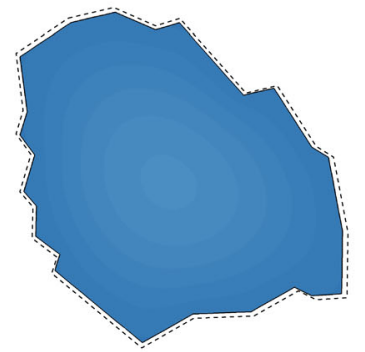

$t=1650 \mathrm{~s}$

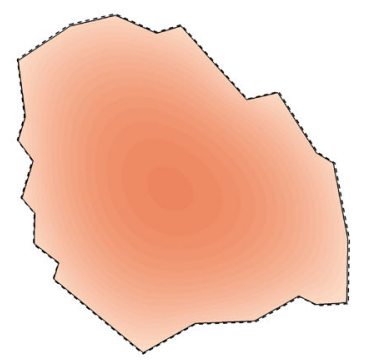

$t=1200 \mathrm{~s}$

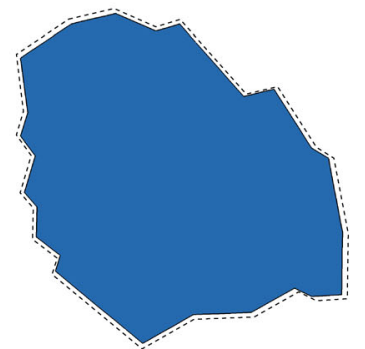

$t=2000 \mathrm{~s}$

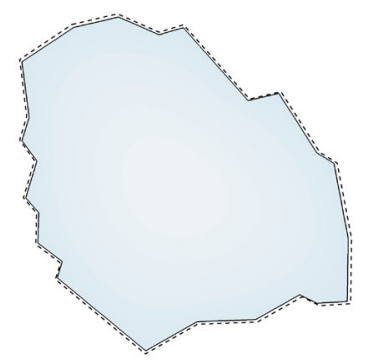

$t=1500 \mathrm{~s}$

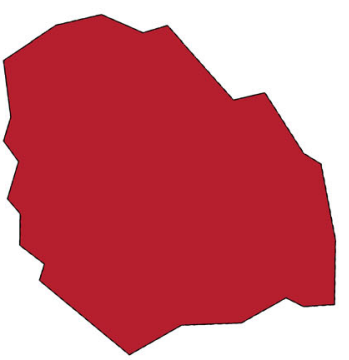

$t=4000 \mathrm{~s}$

c

0.996

Fig. 13 Contour plots of concentration within the particle during the first half of the charging-discharging process at time instances $t=\{0,1100,1200,1500,1550,1650,2000\} \mathrm{s}$ and at the final stage of loading at $t=4000 \mathrm{~s}$. The dashed line represents the undeformed dimensions of the particle

Acknowledgements The financial support of the German Research Foundation (DFG) in the framework of project MI 295/20-1 is gratefully acknowledged.

Funding Open Access funding enabled and organized by Projekt DEAL.

Open Access This article is licensed under a Creative Commons Attribution 4.0 International License, which permits use, sharing, adaptation, distribution and reproduction in any medium or format, as long as you give appropriate credit to the original author(s) and the source, provide a link to the Creative Commons licence, and indicate if changes were made. The images or other third party material in this article are included in the article's Creative Commons licence, unless indicated otherwise in a credit line to the material. If material is not included in the article's Creative Commons licence and your intended use is not permitted by statutory regulation or exceeds the permitted use, you will need to obtain permission directly from the copyright holder. To view a copy of this licence, visit http://creativecommons.org/licenses/by/4.0/.

\section{References}

1. Bai, Y., Zhao, K., Liu, Y., Stein, P., Xu, B.-X.: A chemo-mechanical grain boundary model and its application to understand the damage of Li-ion battery materials. Scripta Mater. 183, 45-49 (2020)

2. Baker, D.R., Verbrugge, M.W.: Temperature and current distribution in thin-film batteries. J. Electrochem. Soc. 146(7), 2413 (1999)

3. Bandhauer, T.M., Garimella, S., Fuller, T.F.: A critical review of thermal issues in lithium-ion batteries. J. Electrochem. Soc. 158(3), R1-R25 (2011)

4. Bernardi, D., Pawlikowski, E., Newman, J.: A general energy balance for battery systems. J. Electrochem. Soc. 132(1), 5-12 (1985)

5. Bohn, E.: Partikel-Modell für Lithium-Diffusion und mechanische Spannungen einer Interkalationselektrode. Ph.D. Thesis, Karlsruher Institute für Technologie (2011)

6. Bohn, E., Eckl, T., Kamlah, M., McMeeking, R.: A model for lithium diffusion and stress generation in an intercalation storage particle with phase change. J. Electrochem. Soc. 160, A1638-A1652 (2013)

7. Bower, A.F., Guduru, P.R., Sethuraman, V.A.: A finite strain model of stress, diffusion, plastic flow, and electrochemical reactions in a lithium-ion half-cell. J. Mech. Phys. Solids 59(4), 804-828 (2011) 
8. Buchmann, I.: Batteries in a Portable World: A Handbook on Rechargeable Batteries for Non-engineers. Cadex Electronics, Richmond (2001)

9. Chen, S., Wan, C., Wang, Y.: Thermal analysis of lithium-ion batteries. J. Power Sources 140(1), 111-124 (2005)

10. Cheng, Y.-T., Verbrugge, M.W.: The influence of surface mechanics on diffusion induced stresses within spherical nanoparticles. Appl. Phys. Lett. 104(083521), 1-6 (2008)

11. Cheng, Y.-T., Verbrugge, M.W.: Evolution of stress within a spherical insertion electrode particle under potentiostatic and galvanostatic operation. J. Power Sources 190, 453-460 (2009)

12. Cheng, Y.-T., Verbrugge, M.W.: Diffusion-induced stress, interfacial charge transfer, and criteria for avoiding crack initiation of electrode particles. J. Electrochem. Soc. 157, A508-A516 (2010)

13. Christensen, J., Newman, J.: Stress generation and fracture in lithium insertion materials. J. Solid State Electrochem. 10, 293-319 (2006)

14. Christensen, J., Newman, J.: A mathematical model of stress generation and fracture in lithium manganese oxide. J. Electrochem. Soc. 153, A1019-A1030 (2006)

15. Dal, H., Miehe, C.: Computational electro-chemo-mechanics of lithium-ion battery electrodes at finite strains. Comput. Mech. 55(2), 303-325 (2015)

16. De Groot, S.R., Mazur, P.: Non-equilibrium Thermodynamics. Dover, New York (1984)

17. Deshpande, R., Cheng, Y.-T., Verbrugge, M.W., Timmons, A.: Diffusion induced stresses and strain energy in a phasetransforming spherical electrode particle. J. Electrochem. Soc. 158(6), A718-A724 (2011)

18. Deshpande, R., Verbrugge, M., Cheng, Y.-T., Wang, J., Liu, P.: Battery cycle life prediction with coupled chemical degradation and fatigue mechanics. J. Electrochem. Soc. 159(10), A1730-A1738 (2012)

19. Deshpande, R.D., Bernardi, D.M.: Modeling solid-electrolyte interphase (SEI) fracture: coupled mechanical/chemical degradation of the lithium ion battery. J. Electrochem. Soc. 164(2), A461-A474 (2017)

20. Diouf, B., Pode, R.: Potential of lithium-ion batteries in renewable energy. Renewable Energy 76, 375-380 (2015)

21. Doyle, M., Fuller, T.F., Newman, J.: Modeling of galvanostatic charge and discharge of the lithium/polymer/insertion cell. J. Electrochem. Soc. 6, 1526-1533 (1993)

22. Doyle, M., Newman, J., Gozdz, A.S., Schmutz, C.N., Tarascon, J.-M.: Comparison of modeling predictions with experimental data from plastic lithium ion cells. J. Electrochem. Soc. 143(6), 1890-1903 (1996)

23. Feng, X., Ouyang, M., Liu, X., Lu, L., Xia, Y., He, X.: Thermal runaway mechanism of lithium ion battery for electric vehicles: a review. Energy Storage Mater. 10, 246-267 (2018)

24. Fuller, T.F., Doyle, M., Newman, J.: Simulation and optimization of the dual lithium ion insertion cell. J. Electrochem. Soc. 141(1), 1-10 (1994)

25. García, R.E., Chiang, Y.-M., Carter, W.C., Limthongkul, P., Bishop, C.M.: Microstructural modeling and design of rechargeable lithium-ion batteries. J. Electrochem. Soc. 152(1), A255-A263 (2005)

26. Golmon, S., Maute, K., Dunn, M.L.: Numerical modeling of electrochemical-mechanical interactions in lithium polymer batteries. Comput. Struct. 87, 1567-1579 (2009)

27. Gu, W., Wang, C.: Thermal-electrochemical modeling of battery systems. J. Electrochem. Soc. 147(8), 2910 (2000)

28. Hu, B., Ma, Z., Lei, W., Zou, Y., Lu, C.: A chemo-mechanical model coupled with thermal effect on the hollow core-shell electrodes in lithium-ion batteries. Theoret. Appl. Mech. Lett. 7(4), 199-206 (2017)

29. Hu, S., Shen, S.: Non-equilibrium thermodynamics and variational principles for fully coupled thermal-mechanical-chemical processes. Acta Mech. 224(12), 2895-2910 (2013)

30. Klinsmann, M., Rosato, D., Kamlah, M., McMeeking, R.M.: Modeling crack growth during Li extraction in storage particles using a fracture phase field approach. J. Electrochem. Soc. 163(2), A102-A118 (2016)

31. Klinsmann, M., Rosato, D., Kamlah, M., McMeeking, R.M.: Modeling crack growth during Li insertion in storage particles using a fracture phase field approach. J. Mech. Phys. Solids 92, 313-344 (2016)

32. Klinsmann, M., Rosato, D., Kamlah, M., McMeeking, R.M.: Modeling crack growth during Li extraction and insertion within the second half cycle. J. Power Sources 331, 32-42 (2016)

33. Latz, A., Zausch, J.: Thermodynamic consistent transport theory of Li-ion batteries. J. Power Sources 196(6), 3296-3302 (2011)

34. Lyu, D., Ren, B., Li, S.: Failure modes and mechanisms for rechargeable Lithium-based batteries: a state-of-the-art review. Acta Mech. 230(3), 701-727 (2019)

35. Masmoudi, M., Moumni, Z., Bidault, F.: Analysis of the thermo-mechanical-chemical coupled response of a lithium-ion battery particle during a charge-discharge cycle. J. Electrochem. Soc. 166(3), A5445-A5461 (2019)

36. Miehe, C., Mauthe, S., Ulmer, H.: Formulation and numerical exploitation of mixed variational principles for coupled problems of Cahn-Hilliard-type and standard diffusion in elastic solids. Int. J. Numer. Meth. Eng. 99(10), 737-762 (2014)

37. Miehe, C., Schänzel, L.-M., Ulmer, H.: Phase field modeling of fracture in multi-physics problems. Part I. Balance of crack surface and failure criteria for brittle crack propagation in thermo-elastic solids. Comput. Methods Appl. Mech. Eng. 294, 449-485 (2015)

38. Miehe, C., Dal, H., Schänzel, L.-M., Raina, A.: A phase-field model for chemo-mechanical induced fracture in lithium-ion battery electrode particles. Int. J. Numer. Meth. Eng. 106(9), 683-711 (2016)

39. Newman, J., Thomas-Alyea, K.E.: Electrochemical Systems. Wiley, New York (2012)

40. Ragavendran, K.R., Mandal, P., Yarlagadda, S.: Correlation between battery material performance and cooperative electronphonon interaction in $\mathrm{LiCo}_{y} \mathrm{Mn}_{2-y} \mathrm{O}_{4}$. Appl. Phys. Lett. 110(14), 143901 (2017)

41. Rahman, M.A., Saghir, M.Z.: Thermodiffusion or Soret effect: historical review. Int. J. Heat Mass Transf. 73, 693-705 (2014)

42. Renganathan, S., Sikha, G., Santhanagopalan, S., White, R.E.: Theoretical analysis of stresses in a lithium ion cell. J. Electrochem. Soc. 157(2), A155-A163 (2010)

43. Singh, G.K., Ceder, G., Bazant, M.Z.: Intercalation dynamics in rechargeable battery materials: general theory and phasetransformation waves in $\mathrm{LiFePO}_{4}$. Electrochim. Acta 53, 7599-7613 (2008) 
44. Tang, M., Huang, H.-Y., Meethong, N., Kao, Y.-H., Carter, W.C., Chiang, Y.-M.: Model for the particle size, overpotential, and strain dependence of phase transition pathways in storage electrodes: application to nanoscale olivines. Chem. Mater. 21(8), 1557-1571 (2009)

45. Thomas, K.E., Newman, J.: Thermal modeling of porous insertion electrodes. J. Electrochem. Soc. 150(2), A176-A192 (2003)

46. Thomas, K.E., Bogatu, C., Newman, J.: Measurement of the entropy of reaction as a function of state of charge in doped and undoped lithium manganese oxide. J. Electrochem. Soc. 148(6), A570-A575 (2001)

47. Verbrugge, M.W., Cheng, Y.-T.: Stress and strain-energy distributions within diffusion-controlled insertion-electrode particles subjected to periodic potential excitations. J. Electrochem. Soc. 156(11), A927-A937 (2009)

48. Wang, D., Wu, X., Wang, Z., Chen, L.: Cracking causing cyclic instability of $\mathrm{LiFePO}_{4}$ cathode material. J. Power Sources 140(1), 125-128 (2005)

49. Wang, H., Jang, Y.-I., Huang, B., Sadoway, D.R., Chiang, Y.-M.: TEM study of electrochemical cycling-induced damage and disorder in $\mathrm{LiCoO}_{2}$ cathodes for rechargeable lithium batteries. J. Electrochem. Soc. 146(2), 473-480 (1999)

50. Weng, L., Xu, C., Chen, B., Zhou, J., Cai, R., Wang, F.: Theoretical analysis of the mechanical behavior in Li-ion battery cylindrical electrodes with phase transformation. Acta Mech. 231(3), 1045-1062 (2020)

51. Werner, M., Weinberg, K.: Coupled thermal and electrochemical diffusion in solid state battery systems. In: New Achievements in Continuum Mechanics and Thermodynamics, pp. 519-535. Springer (2019)

52. Whittingham, M.S.: Lithium batteries and cathode materials. Chem. Rev. 104(10), 4271-4302 (2004)

53. Xing, H., Liu, Y., Wang, B.: Mechano-electrochemical and buckling analysis of composition-gradient nanowires electrodes in lithium-ion battery. Acta Mech. 230(12), 4145-4156 (2019)

54. Yang, H., Amiruddin, S., Bang, H.J., Sun, Y.-K., Prakash, J.: A review of Li-ion cell chemistries and their potential use in hybrid electric vehicles. J. Ind. Eng. Chem. 12(1), 12-38 (2006)

55. Zhang, D., Popov, B.N., White, R.E.: Modeling lithium intercalation of a single spinel particle under potentiodynamic control. J. Electrochem. Soc. 147(3), 831-838 (2000)

56. Zhang, X., Sastry, A.M., Shyy, W.: Numerical simulation of intercalation-induced stress in Li-ion battery electrode particles. J. Electrochem. Soc. 154, A910-A916 (2007)

57. Zhang, X., Sastry, A.M., Shyy, W.: Intercalation-induced stress and heat generation within single lithium-ion battery cathode particles. J. Electrochem. Soc. 155, A542-A552 (2008)

58. Zhao, K., Pharr, M., Cai, S., Vlassak, J.J., Suo, Z.: Large plastic deformation in high-capacity lithium-ion batteries caused by charge and discharge. J. Am. Ceram. Soc. 94, 226-235 (2011)

59. Zhao, Y., Stein, P., Xu, B.-X.: Isogeometric analysis of mechanically coupled Cahn-Hilliard phase segregation in hyperelastic electrodes of Li-ion batteries. Comput. Methods Appl. Mech. Eng. 297, 325-347 (2015)

60. Zhao, Y., Stein, P., Bai, Y., Al-Siraj, M., Yang, Y., Xu, B.-X.: A review on modeling of electro-chemo-mechanics in lithium-ion batteries. J. Power Sources 413, 259-283 (2019)

61. Zubi, G., Dufo-López, R., Carvalho, M., Pasaoglu, G.: The lithium-ion battery: State of the art and future perspectives. Renew. Sustain. Energy Rev. 89, 292-308 (2018)

Publisher's Note Springer Nature remains neutral with regard to jurisdictional claims in published maps and institutional affiliations. 